\title{
LATTICE WESS-ZUMINO-WITTEN MODEL AND QUANTUM GROUPS円
}

\author{
Fernando FALCETOF \\ I.H.E.S., 91440 Bures-sur-Yvette, France \\ Krzysztof GAWȨDZKI \\ C.N.R.S., I.H.E.S., 91440 Bures-sur Yvette, France,
}

\begin{abstract}
Quantum groups play a role of symmetries of integrable theories in two dimensions. They may be detected on the classical level as Poisson-Lie symmetries of the corresponding phase spaces. We discuss specifically the Wess-Zumino-Witten conformally invariant quantum field model combining two chiral parts which describe the left- and right-moving degrees of freedom. On one hand side, the quantum group plays the role of the symmetry of the chiral components of the theory. On the other hand, the model admits a lattice regularization (in the Minkowski space) in which the current algebra symmetry of the theory also becomes quantum, providing the simplest example of a quantum group symmetry coupling space-time and internal degrees of freedom. We develop a free field approach to the representation theory of the lattice $s l(2)$-based current algebra and show how to use it to rigorously construct an exact solution of the quantum $S L(2)$ WZW model on lattice.
\end{abstract}

\section{Introduction}

The relations to two-dimensional quantum field theories and integrable lattice models have been at the origin of quantum groups [1], [2], objects abstracted from the remarkable properties of exactly soluble two-dimensional systems. The language of quantum groups, as that of the standard groups, has become a convenient tool to describe symmetry properties of physical systems. Quantum groups are one-parameter deformations of classical Lie groups or, if we identify the deformation parameter with $\hbar$, their quantization. A more rewarding point of

\footnotetext{
${ }^{1}$ Extended version of lectures given by the second author at the XXVIIIth Karpacz Winter School of Theoretical Physics, February 17 - 29, 1992

${ }^{2}$ Present address: Department of Theoretical Physics, University of Zaragoza, Zaragoza, Spain
} 
view is however the one in which, on the classical level, we retain the information about the infinitesimal direction of the deformation. We obtain then the notion of a Poisson-Lie (PL) group, i.e. a Lie group with a compatible Poisson bracket on it [3], [4].

The standard symmetries of classical systems are described by the action of the symmetry group elements on the phase space of the system in a way which preserves the Poisson brackets of physical quantities. The infinitesimal version of the action of continuous symmetry groups on physical quantities may then be generated by Poisson bracket with hamiltonians whose collection goes under the name of a moment map [5]. Giving the moment map is an alternative way to describe the classical symmetry.

PL groups lead to a generalization of the concept of classical symmetry. One requires that not the individual symmetry transformations but the action of the group viewed as a transformation from phase space $\times$ group into phase space preserves Poisson brackets. The notion of a moment map may be generalized to the case of PL symmetries: the infinitesimal transformations of physical quantities are still generated by Poisson brackets, this time with a non-linear collection of hamiltonians (in terminology advocated in [6]).

When quantizing a classical system with standard symmetries, one often attempts to preserve the symmetries, i.e. to get the action of the same symmetry groups in the quantum space of states in such a way that the interesting families of observables transform in a simple way mimicking their classical covariance properties (this is not always possible). When quantizing a classical system with a PL symmetry, it is natural to demand an action of the corresponding quantum group in the space of states. The covariance properties of the interesting observables should then reduce to the classical expressions in order $\mathcal{O}(\hbar)$. The theory of PL symmetries becomes this way very useful as the means to detect quantum group symmetries of quantum mechanical or quantum field-theoretical models.

The present set of lectures is designed as an illustrative introduction to the subject of Poisson-Lie and quantum group symmetries. We start by a presentation of the elements of the theory of PL groups (Section 2) which may be found in numerous original and review papers, see e.g. [1], [3], [4], [11], [8], [9], [6]. The main body of the exposition discusses an application of those concepts to the Wess-Zumino-Witten (WZW) model of conformal field theory. In Section 3 we describe the canonical formalism of the classical WZW theory following the approach of [10], see also [11-15]. A special stress is put on the details of how the degrees of freedom of the model separate into the left- and right-movers. We show that, besides the standard loop group (or current algebra) and conformal (Virasoro) symmetries, the chiral part of the classical theory posses a finite-dimensional PL symmetry. In Section 4, we demonstrate how the last two symmetries may be separated by decomposing the chiral phase space of the WZW model. This decomposition is the classical counterpart of the vertex-IRF transformation well known from the theory of lattice integrable systems [16 or from the analysis of the quantum WZW theory [17]. The loop group part of the chiral phase space is essentially a union of coadjoint orbits of the infinite-dimensional Kac-Moody group centrally extending the loop group. Similarly, the Poisson-Lie part is composed of PL deformations of the coadjoint orbits for the finite dimensional PL group. Under quantization, they give spaces, respectively, of irreducible representations of Kac-Moody algebra and (Drinfeld-Jimbo [1],[2]) quantum group. The space of 
quantum states of the chiral WZW theory combines both. In Section 5, we describe a free field realization of the loop group part of the chiral WZW phase space for the simplest case of group $S L(2)$, see also [14]. The free fields provide Darboux coordinates on the phase space and a convenient starting point for the Wakimoto-Bernard-Felder approach to the representation theory of $s l(2)$-based Kac-Moody algebra. This approach realizes the irreducible representations of the latter in a cohomology of Fock spaces.

As most quantum theories, the WZW model requires a (field strength) renormalization. This complicates the rigorous construction of the theory which involves fine and interesting mathematical points far from being completely clarified (most members of the conformal physics community are probably not aware of this state of affairs). It is then interesting to realize that the model possesses a lattice regularization which essentially preserves the continuum symmetries of the theories. For the related Liouville and Toda theories such regularizations where discussed in [18] to [22]. For the WZW theory, the lattice regularization may be based on the concept of the lattice Kac-Moody algebra introduced in a series of papers of the LeningradSt.Petersburg group [23]-25]. The regularization may be introduced already on the classical level in such a way that the theory still separates into the left- and right-moving sectors and that the loop group symmetry of the continuum theory becomes a local Poisson-Lie symmetry, as described in Section 6. The classical vertex-IRF transformation permits to separate further the degrees of freedom carrying the local and global PL symmetries. In the quantum theory, the moment map of the local PL symmetry becomes the St. Petersburg lattice Kac-Moody algebra. We present it (for the simplest $s l(2)$ case) in Section 7 . One of its remarkable features is that it implies a lattice version of conformal symmetry formulated as an invariance under a block spin type renormalization group! In fact the lattice Kac-Moody algebra realizes a simplest coupling of the quantum groups to the space-time degrees of freedom and might be thought of as an elementary step towards employing quantum groups as space-time symmetries. The quantum space of states corresponding to the lattice Kac-Moody algebra may be built from special representations of the latter obtained by a deformation of the free field approach à la Wakimoto-Bernard-Felder. This is described in Sections 8 to 10 containing an original material, see also [26]. In the simplest case of one lattice site, the lattice Kac-Moody algebra reduces to the (Drinfeld-Jimbo) quantum group. As a result, by specialization, the free field construction of representations of lattice Kac-Moody algebra provides also a possible approach to the representation theory of the quantum group. We provide its details in Section 11 which may be read independently and/or prior to Sections 8 to 10. Finally, in Section 12, we combine the spaces of states of lattice Kac-Moody and quantum group degrees of freedom and show how dressing of the first ones with the second allows to obtain field operators with braiding given by the quantum $S L(2) R$-matrices in fundamental representation. This provides a lattice realization of the idea spelled out in [17].

As discussed in [27]-29], the continuum chiral WZW model is closely related to the threedimensional topological Chern-Simons gauge theory: it essentially describes the Schrödinger picture of the latter. The main feature of the representation theory of the Kac-Moody algebras which underlies the relation between the WZW and the Chern-Simons models is the existence of the special tensor product of representations of Kac-Moody algebras called fusion. There are 
many indications that there should exist a lattice regularization of the Chern-Simons model, a type of a quantum-group gauge theory, which is still topological (invariant under lattice subdivisions). In fact, the expectation values of the Chern-Simons theory (giving 3-manifold and knot invariants) may be computed as lattice statistical sums involving quantum group objects [30, 31. We expect that the chiral lattice WZW theory described in these lectures may be a possible step towards a lattice Chern-Simons topological quantum group gauge theory. The main missing step seems to be the lattice fusion operation which, up to now, we have under complete control only if one of the fused representations corresponds to the lattice with a single point.

Another open problem, equally interesting, is to find a lattice version of the coset construction which, in continuum, allows to produce a multitude of conformal field theories out from the WZW one. In particular, the lattice version of Drinfeld-Sokolov reduction should reproduce the lattice versions 20] to 22] of Toda theories.

\section{Poisson-Lie symmetry}

\section{2.a. Poisson manifolds}

One of the essential concepts of the classical physics is that of the Poisson bracket of physical quantities. Mathematically, one considers manifolds $M$ with a (smooth) field of 2-vectors $\Pi\left(=\sum_{i, j} \Pi^{i j} \frac{\partial}{\partial x^{i}} \wedge \frac{\partial}{\partial x^{j}}\right.$ in local coordinates $)$ s. t. the Poisson bracket of functions $f, g$ on $M$ given by the contraction of $\Pi$ with 2 -form $d f \wedge d g$ :

$$
\{f, g\}=\langle\Pi, d f \wedge d g\rangle
$$

satisfies the Jacobi identity.

$$
\{\{f, g\}, h\}+\{\{g, h\}, f\}+\{\{h, f\}, g\}=0 .
$$

$\Pi$ is called then a Poisson structure on $M . M$ together with the Poisson bracket is called a Poisson manifold. There are two extreme cases of Poisson structures: $\Pi \equiv 0$ and $\Pi$ nondegenerate, i.e. $\operatorname{det}\left(\Pi^{i j}\right)=0$. In the latter case, where necessarily $M$ is even-dimensional, $\Pi$ induces a symplectic form $\Omega$ on $M$ equal locally to $\sum_{i, j}\left(\Pi^{-1}\right)_{i j} d x^{j} \wedge d x^{i}$. On symplectic manifolds, the only functions which Poisson commute with all others are constants. In general, $M$ may be foliated into (maximal connected) manifolds, called symplectic leaves, on which each function on $M$ Poisson commuting with the others is constant and on which the Poisson structure induces a symplectic form. If $\Pi \equiv 0$, the symplectic leaves are the points of $M$.

For two Poisson manifolds $\left(M_{i}, \Pi_{i}\right), i=1,2, \Pi_{1}+\Pi_{2}$ defines a Poisson structure on $M_{1} \times M_{2}$. If $f_{i}, g_{i}$ are functions on $M_{i}$, respectively, then

$$
\left\{f_{1} f_{2}, g_{1} g_{2}\right\}=\left\{f_{1}, g_{1}\right\} f_{2} g_{2}+f_{1} g_{1}\left\{f_{2}, g_{2}\right\} .
$$

A smooth map $P: M_{1} \rightarrow M_{2}$ is called Poisson if on $P\left(M_{1}\right)$

$$
P_{*} \Pi_{1}=\Pi_{2}
$$


where $P_{*}$ denotes the tangent map. Then, for functions $f, g$ on $M_{2}$,

$$
\left\{P^{*} f, P^{*} g\right\}=P^{*}\{f, g\} .
$$

\section{2.b. Poisson-Lie groups and Lie bialgebras}

Crossbreeding of the concepts of a Poisson manifold and of a Lie group has led to the notion of a Poisson-Lie (PL) group [3], 团. This is a Lie group supplied with a Poisson structure such that the group multiplication map

$$
m: G \times G \mapsto G
$$

is Poisson. Below, we shall work in the complex category, unless otherwise stated. On the infinitesimal level, one obtains from the Poisson structure of $G$ the Lie algebra bracket $[\cdot, \cdot]^{*}$ on the dual space $\mathcal{G}^{*}$ to the Lie algebra $\mathcal{G}$ of $G$ :

$$
[d f(1), d g(1)]^{*}=d\{f, g\}(1) .
$$

The fact that the group multiplication is a Poisson map leads to the following compatibility condition between the Lie brackets of $\mathcal{G}$ and $\mathcal{G}^{*}$ :

$$
\left\langle[X, Y],[v, w]^{*}\right\rangle+\left\langle a d_{v}^{*} X, a d_{Y}^{*} w\right\rangle-\left\langle a d_{w}^{*} X, a d_{Y}^{*} v\right\rangle-\left\langle a d_{v}^{*} Y, a d_{X}^{*} w\right\rangle+\left\langle a d_{w}^{*} Y, a d_{X}^{*} v\right\rangle=0 .
$$

By dualization, $[\cdot, \cdot]^{*}$ induces a map

$$
c: \mathcal{G} \rightarrow \mathcal{G} \wedge \mathcal{G} .
$$

In terms of $c$, eq. (8) becomes the cocycle condition

$$
c[X, Y]-a d_{X} c(Y)+a d_{Y} c(X)=0 .
$$

A pair of finite-dimensional Lie algebras $\left(\mathcal{G}, \mathcal{G}^{*}\right)$ with Lie brackets satisfying (8) is called a Lie bialgebra. Each Lie bialgebra corresponds to a unique connected, simply connected PL group. Because $\mathcal{G}$ and $\mathcal{G}^{*}$ enter symmetrically into $(8),\left(\mathcal{G}^{*}, \mathcal{G}\right)$ is also a Lie bialgebra which we shall call dual to $\left(\mathcal{G}, \mathcal{G}^{*}\right)$. The PL group $\left(G^{*},\{\cdot, \cdot\}^{*}\right)$ corresponding to $\left(\mathcal{G}^{*}, \mathcal{G}\right)$ will be called dual to $(G,\{\cdot, \cdot\})$. Thus (connected, simply connected) PL groups come in dual pairs.

$\mathcal{G}$ and $\mathcal{G}^{*}$ may be put as Lie subalgebras into the double Lie algebra $\widetilde{\mathcal{G}}$, equal $\mathcal{G} \oplus \mathcal{G}^{*}$ as the vector space, with the Lie bracket

$$
[X+v, Y+w] \equiv[X, Y]+[v, w]^{*}-a d_{X}^{*} w+a d_{Y}^{*} v+a d_{w}^{*} X-a d_{v}^{*} Y .
$$

They form maximal isotropic subspaces of $\widetilde{\mathcal{G}}$ with respect to ad-invariant non-degenerate bilinear form

$$
(X+v, Y+w) \equiv\langle X, w\rangle+\langle Y, v\rangle .
$$

The (simply connected) group $\widetilde{G}$ corresponding to $\widetilde{\mathcal{G}}$ is the Drinfeld double of PL group $(G,\{.,\}$.$) .$

Conversely, any Lie algebra $\widetilde{\mathcal{G}}$ with a non-degenerate $a d$-invariant bilinear form and a pair of maximal isotropic subalgebras (a Manin triple) gives (a pair of dual) Lie bialgebras by identifying one of the subalgebras with the dual of the other by means of the bilinear form. 


\section{2.c. Examples of PL groups}

Each Lie group may be considered as a PL group if we take $\{\cdot, \cdot\} \equiv 0$. The corresponding Lie bialgebra has trivial bracket $[\cdot, \cdot]^{*} \equiv 0$ on $\mathcal{G}^{*}$. The dual PL group is $\mathcal{G}^{*}$ itself viewed as the additive group together with the Poisson bracket

$$
\{X, Y\}^{*}=[X, Y]
$$

where $X, Y,[X, Y] \in \mathcal{G}$ are viewed as linear functions on $\mathcal{G}^{*}$. The symplectic leaves of $\mathcal{G}^{*}$ are the (connected components of) coadjoint orbits of $G$. They are models of symplectic homogeneous spaces of $G$ and they give rise, by quantization, to irreducible representations of $G$ [32].

An (almost) general example of a PL group is provided by the following construction. Let $r=\sum_{\alpha} X_{\alpha} \otimes Y_{\alpha} \in \wedge^{2} \mathcal{G}$. Define a bracket on $\mathcal{G}^{*}$ by

$$
\left\langle X,[v, w]^{*}\right\rangle=\left\langle a d_{X} r, v \otimes w\right\rangle .
$$

The corresponding cocycle $c: \mathcal{G} \rightarrow \mathcal{G} \wedge \mathcal{G}$ is given by

$$
c(X)=a d_{X} r
$$

i.e. is a coboundary. Bracket $[\cdot, \cdot]^{*}$ satisfies the Jacobi identity if and only if

$$
a d_{X} r_{123}=0
$$

for each $X \in \mathcal{G}$ where

$$
r_{123}=\left[r_{12}, r_{13}\right]+\left[r_{12}, r_{23}\right]+\left[r_{13}, r_{23}\right] J \in \mathcal{G}^{\wedge 3}
$$

and

$$
\left[r_{12}, r_{13}\right]=\sum_{\alpha, \beta}\left[X_{\alpha}, X_{\beta}\right] \otimes Y_{\alpha} \otimes Y_{\beta} \in \mathcal{G}^{\otimes 3}, \quad \text { etc. }
$$

Suppose that $\mathcal{G}$ is a (complex) simple Lie algebra, with the Killing form $\operatorname{tr}$ and a basis $\left(t^{a}\right)$ normalized so that $\operatorname{tr} t^{a} t^{b}=\frac{1}{2} \delta^{a b}$. Any ad-invariant element of $\mathcal{G}^{\wedge 3}$ is proportional to $F=\sum_{a, b, c} f^{a b c} t^{a} \otimes t^{b} \otimes t^{c}$ where $\left[t^{a}, t^{b}\right]=\sum_{c} f^{a b c} t^{c}$, so that $r_{123} \sim F$ or, equivalently, denoting the proportionality constant as $-\left(\frac{2 \pi}{k}\right)^{2}$ for later convenience,

$$
r_{123}^{ \pm}=0
$$

where

$$
r^{ \pm} \equiv r \pm \frac{2 \pi}{k} C
$$

with the quadratic Casimir $C=\sum_{a} t^{a} \otimes t^{a}$. Condition (15) is known as the classical Yang-Baxter (CYB) equation. A possible choice for $r$ is

$$
r=\frac{\pi}{k} \sum_{\alpha>0}\left(e_{\alpha} \otimes e_{-\alpha}-e_{-\alpha} \otimes e_{\alpha}\right)
$$


where $e_{\alpha}$ are the step generators of $\mathcal{G}$ corresponding to roots $\alpha$. We shall refer to the corresponding $r^{ \pm}$as the standard solutions of the CYB equation. In the case of simple $\mathcal{G}$ (and $\frac{2 \pi}{k} \neq 0$ ) one may realize $\mathcal{G}^{*}$ with its Lie bracket (11) as a subalgebra of $\mathcal{G} \oplus \mathcal{G}$ by the embedding

$$
\mathcal{G}^{*} \ni v \mapsto\left(\iota(v) r^{+}, \iota(v) r^{-}\right) \in \mathcal{G} \oplus \mathcal{G}
$$

which is a Lie algebra homomorphism due to the CYB equation (14). $\iota(v) r^{ \pm}$denotes the contraction of $v$ with the first component of $r^{ \pm}$. For the standard $r$-matrix (17), one obtains this way the subgroup of $b^{-} \oplus b^{+}\left(b^{ \pm}\right.$are the Borel subgroups of $\left.\mathcal{G}\right)$ composed of elements with opposite Cartan subalgebra components.

The Poisson bracket of the PL group $(G,\{.,\}.) \equiv G_{r}$ corresponding to Lie bialgebra $\left(\mathcal{G}, \mathcal{G}^{*}\right)$ with bracket (11) may be described the following way. Let $g$ denote the matrix of functions on $G$ given by a finite-dimensional representation of $G$. Let $g_{1}=g \otimes 1, g_{2}=1 \otimes g$ where 1 is the unit matrix. Then the formula

$$
\left\{g_{1}, g_{2}\right\}=\left[g_{1} g_{2}, r\right]\left(=\left[g_{1} g_{2}, r^{ \pm}\right]\right)
$$

where $r$ is taken in the same representation, gives the Poisson bracket of all matrix elements of $g$. For simple $\mathcal{G}$, the dual PL group $G_{r}^{*}$ may be realized as a subgroup of $G \times G$. For the standard $r$-matrix one obtains the subgroup of $B^{-} \times B^{+} \quad\left(B^{ \pm}\right.$are the Borel subgroups) composed of elements $\left(\gamma^{-}, \gamma^{+}\right)=\left(n^{-} t, n^{+} t^{-1}\right)$ where $n^{ \pm}$are in the nilpotent subgroups and $t$ is in the Cartan subgroup $T$ of $G$. The Poisson bracket $\{\cdot, \cdot\}^{*}$ on $G^{*}$ is given by the formulae

$$
\begin{aligned}
& \left\{\gamma_{1}^{+}, \gamma_{2}^{+}\right\}^{*}=\left[r, \gamma_{1}^{+} \gamma_{2}^{+}\right] \\
& \left\{\gamma_{1}^{+}, \gamma_{2}^{-}\right\}^{*}=\left[r^{+}, \gamma_{1}^{+} \gamma_{2}^{-}\right], \\
& \left\{\gamma_{1}^{-}, \gamma_{2}^{-}\right\}^{*}=\left[r, \gamma_{1}^{-} \gamma_{2}^{-}\right] .
\end{aligned}
$$

By the map $\left(\gamma^{-}, \gamma^{+}\right) \mapsto \gamma^{-}\left(\gamma^{+}\right)^{-1}=\gamma, G^{*}$ covers finitely many times an open subset of $G$ (which, for the standard $r$-matrix is also dense). The Poisson bracket $\{\cdot, \cdot\}^{*}$

$$
\left\{\gamma_{1}, \gamma_{2}\right\}^{*}=r^{+} \gamma_{1} \gamma_{2}-\gamma_{1} r^{+} \gamma_{2}-\gamma_{2} r^{-} \gamma_{1}+\gamma_{1} \gamma_{2} r^{-}
$$

makes the covering map Poisson. (The Poisson bracket $\{\cdot, \cdot\}^{*}$, as contrasted with the one of (19) does not make $G$ a PL group). The conjugacy classes in $G$ form the symplectic leaves of $\left(G,\{\cdot,\}^{*}\right)$. The connected components of the $\chi$-preimages of the conjugacy classes form the symplectic leaves of $G^{*}$ [4] which play in the PL category the role generalizing that of the coadjoint orbits in the Lie category. In particular, for standard $r$-matrix, their quantization gives the irreducible representations of quantum group $\mathcal{U}_{q}(\mathcal{G})$ deforming the universal enveloping algebra of $\mathcal{G}$.

\section{2.d. Moment maps}

Let $(M,\{\cdot, \cdot\})$ be a Poisson manifold. We say that Lie group $G$ is a symmetry of $M$ if $G$ acts on $M$ (say, from the right) by transformations preserving the Poisson bracket. If $X \in \mathcal{G}$, 
it generates then a vector field $\widetilde{X}$ on $M$. We shall call $\widetilde{X}$ hamiltonian if there exists a function $h_{X}$ s. t.

$$
\widetilde{X}(f)=\left\{h_{X}, f\right\}
$$

for any smooth function $f$ on $M$. If $M$ is symplectic and simply connected then $h_{X}$ always exists. If $\widetilde{X}$ and $\widetilde{Y}$ are hamiltonian then so is $a X+b Y$ and $[\widetilde{X, Y]}$ and we may take

$$
\begin{gathered}
h_{a X+b Y}=a h_{X}+b h_{Y}, \\
h_{[X, Y]}=\left\{h_{X}, h_{Y}\right\} .
\end{gathered}
$$

The action of $G$ is called hamiltonian if each $\widetilde{X}$ is hamiltonian. The hamiltonians $h_{X}$ may be chosen so that (23) is satisfied. Then we obtain a map

$$
m: M \rightarrow \mathcal{G}^{*}
$$

s. t. $\langle X, m(\cdot)\rangle=h_{X}$. If additionally $(24)$ is satisfied, $m$ is a Poisson map if we provide $\mathcal{G}^{*}$ with Poisson bracket (10). In this case $m$ is called the moment map for the action of $G$ on $M$ [5]. If $\mathcal{G}$ is simple, (24) may be always assured. In general, one has to pass to a central extension of $G$ and $\mathcal{G}$.

The notion of a symmetry may be naturally generalized to the PL category. We shall say that a PL group $(G,\{\cdot, \cdot\})$ is a PL symmetry of the Poisson manifold $(M,\{\cdot, \cdot\})$ if the map $M \times G \rightarrow M$ giving the action of $G$ on $M$ is Poisson. If the Poisson bracket on $G$ is trivial, this reduces to the previous notion of the symmetry. We shall call a PL symmetry hamiltonian if there exists a map

$$
m: M \rightarrow G^{*}
$$

s.t.

$$
\widetilde{X}(f)=\left\langle X,\{m, f\} m^{-1}\right\rangle
$$

for each smooth function $f$ on $M\left(\{m, f\} m^{-1} \equiv\left\langle\Pi,(d m) m^{-1} \wedge d f\right\rangle\right.$ where $\Pi$ is the Poisson structure on $M$ ). If $M$ is symplectic and simply connected, then each PL symmetry is hamiltonian [9]. We shall say that $m$ is a moment map if, additionally, it is a Poisson map. Its existence is not guaranteed even for hamiltonian symmetry. In general, $m$ is Poisson only for a modified Poisson structure of $G^{*}$ [9]. Clearly, the moment map allows to reconstruct the (infinitesimal) action of $G$ on $M$.

\section{Classical Wess-Zumino-Witten theory}

The Wess-Zumino-Witten (WZW) theory is an example of a two-dimensional sigma model with fields $g\left(x^{0}, x^{1}\right)$ taking values in the group manifold $G$. The classical equations are

$$
\partial_{-}\left(g \partial_{+} g^{-1}\right)=0
$$

where $\partial_{ \pm}$are the derivatives with respect to the light-cone variables

$$
x^{ \pm}=x^{1} \pm x^{0}
$$


We shall consider the theory on the cylinder obtained by taking $x^{1}$ modulo $2 \pi$. The general solutions of (1) are

$$
g\left(x^{0}, x^{1}\right)=g_{L}\left(x^{+}\right) g_{R}\left(x^{-}\right)^{-1}
$$

where

$$
g_{L, R}\left(x^{ \pm}+2 \pi\right)=g_{L, R}\left(x^{ \pm}\right) \gamma
$$

with monodromy $\gamma \in G$. $\left(g_{L}, g_{R}\right)$ and $\left(g_{L} g, g_{R} g\right)$ with constant $g$ give the same solution. If we introduce spaces $P_{L, R}=\left\{g_{L, R}: \mathbf{R} \rightarrow G \mid g_{L, R}(x+2 \pi)=g_{L, R}(x) \gamma_{L, R}\right\}$, then the phase space $P$ of solutions of (1) becomes

$$
P=\Delta / G
$$

where $\Delta$ is the submanifold of $P_{L} \times P_{R}$ where $\gamma_{L}=\gamma_{R} . P_{L, R}$ will play the role of phase spaces for the left-, right-moving degrees of freedom.

Eq. (1) comes from the Lagrangian

$$
S(g)=\frac{k}{4 \pi} \int \operatorname{tr}\left(g^{-1} \partial_{+} g\right)\left(g^{-1} \partial_{-} g\right) d x^{+} \wedge d x^{-}+\frac{k}{12 \pi} \int d^{-1} \operatorname{tr}\left(g^{-1} d g\right)^{\wedge 3}
$$

( $k$ is the coupling constant) which induces the canonical Poisson bracket on $P$ :

$$
\begin{aligned}
& \left\{g(0, x)_{1}, g(0, y)\right\}=0 \\
& \left\{g(0, x)_{1}, \xi^{0}(0, y)_{2}\right\}=-\frac{8 \pi}{k} g(0, x)_{1} C \delta(x-y) \\
& \left\{\xi^{0}(0, x)_{1}, \xi^{0}(0, y)_{2}\right\}=\frac{8 \pi}{k}\left[C, \xi^{0}(0, x)_{1}+g^{-1} \partial_{1} g(0, x)_{1}\right] \delta(x-y)
\end{aligned}
$$

where $\xi^{0} \equiv g^{-1} \partial_{0} g$. The corresponding symplecting form is

$$
\Omega=\frac{k}{4 \pi} \int_{0}^{2 \pi} \operatorname{tr}\left[-d \xi^{0} \wedge\left(g^{-1} d g\right)+\left(\xi^{0}+g^{-1} \partial_{1} g\right)\left(g^{-1} d g\right)^{\wedge 2}\right](0, x) .
$$

Rewriting $\Omega$ in terms of $g_{L, R}$, we obtain

$$
\Omega(g)=\Omega_{L}\left(g_{L}\right)-\Omega_{R}\left(g_{R}\right)
$$

where

$$
\begin{array}{r}
\Omega_{L}\left(g_{L}\right)=\frac{k}{4 \pi} \int_{0}^{2 \pi} \operatorname{tr}\left(g_{L}^{-1} d g_{L}\right) \wedge \partial\left(g_{L}^{-1} d g_{L}\right) \\
+\frac{k}{4 \pi} \operatorname{tr} g_{L}^{-1} d g_{L}(0) \wedge\left(d \gamma_{L}\right) \gamma_{L}^{-1}-\frac{k}{4 \pi} \rho\left(\gamma_{L}\right)
\end{array}
$$

and $\Omega_{R}$ is given by the same formula with $g_{R} \rightarrow g_{L} . \quad \rho$ is an arbitrary 2-form on $G$. The ambiguity is due to the fact that $\Omega$ is given only on the subset $\Delta$ of $P_{L} \times P_{R}$. Since

$$
d \Omega_{L}\left(g_{L}\right)=\frac{k}{12 \pi} \operatorname{tr}\left(\gamma_{L}^{-1} d \gamma_{L}\right)^{\wedge 3}-\frac{k}{4 \pi} d \rho\left(\gamma_{L}\right)
$$

and $\operatorname{tr}\left(\gamma^{-1} d \gamma\right)^{\wedge 3}$ is a closed but not exact form on (simple) $G, \Omega_{L}$ is never globally closed. One may however choose singular $\rho$ s. t. $d \rho=\frac{1}{3} \operatorname{tr}\left(\gamma^{-1} d \gamma\right)^{\wedge 3}$ on an open (dense) set. It will be convenient to take

$$
\rho(\gamma)=\operatorname{tr}\left(\gamma^{-}\right)^{-1}\left(d \gamma^{-}\right) \wedge\left(\gamma^{+}\right)^{-1}\left(d \gamma^{+}\right)
$$


in terms of the decomposition $\gamma=\gamma^{-}\left(\gamma^{+}\right)^{-1}$ induced by a classical $r$-matrix. This choice leads to the following, globally defined, Poisson bracket on $P_{L}$ :

$$
\begin{aligned}
& \left\{g_{L}(x)_{1}, g_{L}(y)_{2}\right\}=g_{L}(x)_{1} g_{L}(y)_{2} r^{ \pm} \\
& \left\{g_{L}(x)_{1}, \gamma_{L 2}\right\}=g_{L}(x)_{1} \gamma_{L 2} r^{-}-g_{L}(x)_{1} r^{+} \gamma_{L 2}, \\
& \left\{\gamma_{L 1}, \gamma_{L 2}\right\}
\end{aligned}
$$

where in (13) the upper sign in $r^{ \pm}$applies when $x>y$ and the lower one when $x<y$, $|x-y|<2 \pi$. The left-moving phase space $P_{L}$ equipped with the Poisson bracket (13) - (15) possesses a rich symmetry structure.

Conformal symmetry

Let $\widetilde{\mathrm{Diff}}+S^{1}$ denote the group of increasing maps $D: \mathbf{R} \rightarrow \mathbf{R}$ s. t. $D(x+2 \pi)=D(x)+2 \pi$. $\widetilde{\text { Diff }_{+}} S^{1}$ is a central extension by its subgroup $\mathbf{Z}$ of integer shifts of group Diff $+S^{1}$ of orientation preserving diffeomorphisms of $S^{1}$ :

$$
0 \rightarrow \mathbf{Z} \rightarrow \widetilde{\operatorname{Diff}_{+}} S^{1} \rightarrow \operatorname{Diff}_{+} S^{1} \rightarrow 1
$$

$\widetilde{\operatorname{Diff}}_{+} S^{1}$ acts on $P_{L}$ by

$$
g_{L} \mapsto g_{L} \circ D
$$

preserving the Poisson brackets (13) - (15). The moment map is given by the energy momentum tensor

$$
T_{L}(x)=\frac{k}{4 \pi} \operatorname{tr}\left(g_{L}^{-1} \partial_{x} g_{L}\right)^{2}(d x)^{2}
$$

defining, as a quadratic differential, an element dual to $\operatorname{Vect}\left(S^{1}\right)$, i.e. to the vector fields on the circle $\mathbf{R} /(2 \pi \mathbf{Z})$ which form the Lie algebra of $\widetilde{\operatorname{Diff}_{+}} S^{1}$. The $\widetilde{\operatorname{Diff}_{+}} S^{1}$ symmetry of $P_{L}$ (and another copy of it for $\left.P_{R}\right)$ give rise to the action on $P$ of the group $\left(\widetilde{\operatorname{Diff}_{+}} S^{1} \times \widetilde{\operatorname{Diff}_{+}} S^{1}\right) / \mathbf{Z}$ of conformal symmetries of the cylinder (the quotient subgroup is that of the diagonal shifts).

Loop group symmetry

Another symmetry of $P_{L}$ is given by the action of the loop group $L G$ of smooth maps $h: \mathbf{R} \rightarrow G, h(x+2 \pi)=h(x)$, by

$$
g_{L} \mapsto h^{-1} g_{L}
$$

This action is hamiltonian and the currents

$$
J(x)=\frac{k}{2 \pi} g_{L} \partial g_{L}^{-1} d x
$$

give the corresponding map of $P_{L}$ into the space $L \mathcal{G}^{*}$ dual to the Lie algebra $L \mathcal{G}$ of $L G$. This map is, however, not Poisson due to the $\delta^{\prime}$ term in the Poisson bracket

$$
\left\{J(x)_{1}, J(y)_{2}\right\}=2\left[C, J(x)_{1}\right] \delta(x-y)-\frac{k}{\pi} C \delta^{\prime}(x-y) .
$$

We may however pass to the central extension of $L \mathcal{G}$

$$
0 \rightarrow \mathbf{C} \rightarrow \hat{L \mathcal{G}} \rightarrow L \mathcal{G} \rightarrow 0
$$


equal $L \mathcal{G} \oplus \mathbf{C}$ as a vector space where $\mathbf{C}$ is central and

$$
[X, Y](x)=[X(x), Y(x)]+\frac{i}{2 \pi} \int_{0}^{2 \pi} \operatorname{tr} X^{\prime} Y
$$

for $X, Y \in L \mathcal{G} \subset \hat{L \mathcal{G}} . \quad \hat{L \mathcal{G}}$ is the Kac-Moody algebra. On a global level, one obtains the Kac-Moody group $\hat{L G}$ giving the central extension of $L G$

$$
1 \rightarrow \mathbf{C}^{*} \rightarrow \hat{L G} \rightarrow L G \rightarrow 1
$$

$J+{ }_{i} k \in L \mathcal{G}^{*} \oplus \mathbf{C} \cong \hat{L \mathcal{G}^{*}}$ defines then the moment map for the $\hat{L G}$ symmetry of $P_{L}$ (with the center of $\hat{L G}$ acting trivially). On the complete phase space we obtain the $\hat{L G} \times \hat{L G}$ symmetry.

$\underline{G_{r} \text { Poisson-Lie symmetry }}$

The conformal and loop-group symmetries of $P_{L}$ are the standard ones: the action of group elements preserves the Poisson bracket on $P_{L}$. $P_{L}$ possesses also a Poisson-Lie symmetry given by the action of $G$

$$
\left(g_{L}, g\right) \mapsto g_{L} g
$$

which becomes a Poisson map if we consider $G$ as a $P L$ group equipped with Poisson bracket (2.19). The monodromy map

$$
g_{L} \mapsto \gamma
$$

plays essentially the role of the moment map for this action. More exactly, its local lifts to $G^{*} \subset G \times G$

$$
g_{L} \mapsto\left(\gamma^{-}, \gamma^{+}\right)
$$

s. t. $\gamma=\gamma^{-}\left(\gamma^{+}\right)^{-1}$ satisfy the following relations

$$
\begin{aligned}
& \left\{\gamma_{1}^{+}, g_{L}(x)_{2}\right\}=-g_{L}(x)_{2} r^{+} \gamma_{1}^{+}, \\
& \left\{\gamma_{1}^{-}, g_{L}(x)_{2}\right\}=-g_{L}(x)_{2} r^{-} \gamma_{1}^{-}, \\
& \left\{\gamma_{1}^{+}, \gamma_{2}\right\}=\left[r^{+}, \gamma_{2}\right] \gamma_{1}^{+}, \\
& \left\{\gamma_{1}^{-}, \gamma_{2}\right\}=\left[r^{-}, \gamma_{2}\right] \gamma_{1}^{-} .
\end{aligned}
$$

These are equivalent to the defining relation (2.25) of the moment map.

\section{Classical vertex-IRF transformation}

On the open dense subset $P_{L 0}$ of $P_{L}$ composed of $g_{L}$ with monodromies $\gamma$ in the conjugacy classes of elements of the Cartan subgroup $T \subset G$, we may use the following parametrization

$$
g_{L}(x)=h(x) \mathrm{e}^{i \tau x} g_{0}^{-1}
$$

where $h \in L G, \tau \in t$ (the Cartan algebra) and $g_{0} \in G$. Notice that

$$
\gamma=g_{0} \mathrm{e}^{2 \pi i \tau} g_{0}^{-1}
$$


Parametrization (1) defines a map from $L G \times t \times G_{0}$ onto $P_{L_{0}}$. Let $Q^{\vee} \subset t$ denote the coroot lattice and $N(T)$ the normalizer of $T$ in $G$. We shall assume $G$ simply connected. A semi-direct product $N(T) \ltimes Q^{\vee}$ acts on $L G \times t \times G_{0}$ by

$$
\left(h(x), \tau, g_{0}\right) \mapsto\left(h(x) \mathrm{e}^{i q x} w, w^{-1}(\tau-q) w, g_{0} w\right)
$$

for $w \in N(T), q \in Q^{\vee}$. Its orbits describe exactly the ambiguity of parametrization (1). In terms of (1), the 2 -form $\Omega_{L}$ of (3.10) becomes

$$
\Omega_{L}\left(g_{L}\right)=\Omega_{L 1}(h, \tau)+\Omega_{L 2}\left(g_{0}, \tau\right)
$$

where

$$
\Omega_{L 1}(h, \tau)=\frac{k}{4 \pi} \int_{0}^{2 \pi} \operatorname{tr}\left[\left(h^{-1} d h\right) \wedge \partial\left(h^{-1} d h\right)+2 i \tau\left(h^{-1} d h\right)^{\wedge 2}-2 i d \tau \wedge h^{-1} d h\right]
$$

and

$$
\Omega_{L 2}\left(g_{0}, \tau\right)=\frac{k}{4 \pi} \operatorname{tr}\left(g_{0}^{-1} d g_{0}\right) \wedge \mathrm{e}^{2 \pi i \tau}\left(g_{0}^{-1} d g_{0}\right) \mathrm{e}^{-2 \pi i \tau}+k i \operatorname{tr} d \tau \wedge g_{0}^{-1} d g_{0}-\frac{k}{4 \pi} \rho\left(g_{0} \mathrm{e}^{2 \pi i \tau} g_{0}^{-1}\right) .
$$

$\Omega_{L 1}$ and $\Omega_{L 2}$ are closed forms (we could also subtract from $\Omega_{L 1}$ an arbitrary closed 2-form dependent on $\tau$ and add it to $\Omega_{L 2}$ ). Space $M_{L \hat{L G}} \equiv L G \times t$ with symplectic form $\Omega_{L 1}$ is (a covering) of what is called a model space for the Kac-Moody group. When restricted to fixed $\tau \in t, \Omega_{L 1}$ coincides with the pullback to $L G$ of the canonical symplectic form on the coadjoint orbit

$$
\left\{h(i \tau) h^{-1}+h \partial h^{-1}+\frac{1}{i} k \mid h \in L G\right\} \subset \hat{L \mathcal{G}^{*}}
$$

$\tau, \tau^{\prime}$ correspond to the same coadjoint orbit if and only if $\tau^{\prime}=w(\tau+q) w^{-1}$ for $q \in Q^{\vee}$ and $w \in N(T)$, i.e. when $\tau$ and $\tau^{\prime}$ are related by the affine Weyl group. Thus the coadjoint orbits appear in $M_{\hat{L G}}$ with multiplicity given by the affine Weyl group. Similarly, $M_{G_{r}} \equiv G \times t$ plays the role of the (covering) of the model space for the PL group $G_{r}$ with Poisson bracket (2.19). For fixed $\tau \in t, \Omega_{L 2}$ coincides with the pullback to $G$ of the symplectic form on the conjugacy class

$$
\left\{g_{0} \mathrm{e}^{2 \pi i \tau} g_{0}^{-1} \mid g_{0} \in G\right\}
$$

which is a symplectic leaf of the Poisson structure (2.21). We remind that the latter corresponds to that of the dual $P L$ group $G_{r}^{*}$ under the map $\left(\gamma^{-}, \gamma^{+}\right) \mapsto \gamma^{-}\left(\gamma^{+}\right)^{-1}$. Again, $\tau$ and $\tau^{\prime}$ give the same conjugacy class if and only if they are related by the affine Weyl group. Let $\delta \subset M_{\hat{L G}} \times M_{G_{r}}$ denote the submanifold given by equating the $t$ coordinates. Then $P_{L 0}=$ $\delta / N(T) \ltimes Q^{\vee}$. As we see, $P_{L 0}$ may be foliated by leaves being products of coadjoint orbits of $\hat{L G}$ and conjugacy classes of $G$ corresponding to the same $\tau$, each such pair appearing only once.

For the compact real form of group $G$, model space $M_{\hat{L G}}$ may be quantized geometrically. The resulting space of states is (if we translate the original $k$ to $k+\check{h}$ where $\check{h}$ is the dual Coxeter number and use the half-form geometric quantization)

$$
\mathcal{H}_{\hat{L G}}=\bigoplus_{\text {integrable } \lambda} \mathcal{H}_{k, \lambda}
$$


$\mathcal{H}_{k, \lambda}$ is the space of the irreducible unitary representation of $\hat{L G}$ of level $k$ and highest weight $\lambda$ coming from geometric quantization of the coadjoint orbit corresponding to $\tau=(k+h)^{-1}(\lambda+\rho)$. $\rho \equiv \sum_{\alpha>0} \alpha / 2$ is the Weyl vector. Fields $h_{L}(x)$ become the vertex operators (in the fundamental representation) [33.

One may attempt a quantization of model space $M_{G_{r}}$ along similar lines obtaining space

$$
\mathcal{V}=\bigoplus_{\text {integrable } \lambda} \mathcal{V}_{q, \lambda}
$$

where $\mathcal{V}_{q, \lambda}$ is the space of irreducible representation of quantum group $\mathcal{U}_{q}(\mathcal{G})$ for $q=\mathrm{e}^{\pi i /(k+\check{h})}$ of highest weight $\lambda$ coming from the quantization of the symplectic leaf of $G_{r}^{*}$ corresponding to the conjugacy class of $\mathrm{e}^{2 \pi i(k+\tilde{h})^{-1}(\lambda+\rho)}$. Quantum fields $g_{0}$ become the Clebsch-Gordan coefficients of $\mathcal{U}_{q}(\mathcal{G})$ (quantum $3 j$-symbols). The space of states of the chiral WZW model quantizing the phase space $P_{L}$ would then be given by the sum of diagonal products

$$
\bigoplus_{\text {integrable } \lambda} \mathcal{H}_{k, \lambda} \otimes \mathcal{V}_{q, \lambda} \text {. }
$$

Below, we shall realize the quantization program for the lattice regularization of the WZW model using free field representation of the theory rather than geometric quantization.

\section{Free field realization of the WZW model}

Model space $M_{\hat{L} G^{\prime}}$ may be realized in terms of free fields which provide Darboux coordinates for it. Let us describe the construction for the case $G=S L(2, \mathbf{C})$. Parametrize:

$$
h_{L}(x)=\left(\begin{array}{cc}
1 & v(x) \\
0 & 1
\end{array}\right)\left(\begin{array}{cc}
1 & 0 \\
w(x) & 1
\end{array}\right)\left(\begin{array}{cc}
\mathrm{e}^{i \phi(x)} & 0 \\
0 & \mathrm{e}^{-i \phi(x)}
\end{array}\right)
$$

where $v$ and $w$ are periodic and

$$
\phi(x+2 \pi)=\phi(x)+2 \pi p
$$

$\left(\tau=p \sigma_{3}\right)$. Put

$$
\beta=v, \quad \gamma=\mathrm{e}^{-2 i \phi} \partial\left(\mathrm{e}^{2 i \phi} w\right)=\partial w+2 i(\partial \phi) w
$$

$w$ may be recovered from $\phi$ and $\gamma$ :

$$
w(x)=\left(\mathrm{e}^{4 \pi i p}-1\right)^{-1} \mathrm{e}^{-2 i \phi(x)} \int_{x}^{x+2 \pi} \gamma(y) \mathrm{e}^{2 i \phi(y)} d y .
$$

In terms of $\phi, \beta$ and $\gamma$.

$$
\Omega_{L 1}(h, \tau)=\frac{k}{2 \pi} \int d \beta \wedge d \gamma-\frac{k}{2 \pi} \int d \phi \wedge \partial(d \phi)+k d p \wedge d \phi(0),
$$


so that the nontrivial Poisson brackets are

$$
\begin{aligned}
& \{\phi(x), \phi(y)\}=\frac{\pi}{2 k} \operatorname{sgn}(y-x), \\
& \{\phi(x), p\}=\frac{1}{2 k}, \\
& \{\gamma(x), \beta(y)\}=\frac{2 \pi}{k} \delta(x-y) .
\end{aligned}
$$

For the fields, we obtain

$$
h_{L}(x)=\left(\begin{array}{cc}
\left(\Pi-\Pi^{-1}\right) \Psi(x)+\beta(x) \Psi(x)^{-1} Q(x) & \beta(x) \Psi(x)^{-1} \\
\Psi(x)^{-1} Q(x) & \Psi(x)^{-1}
\end{array}\right)\left(\begin{array}{cc}
\left(\Pi-\Pi^{-1}\right)^{-1} & 0 \\
0 & 1
\end{array}\right)
$$

where $\Pi \equiv \mathrm{e}^{2 \pi i p}, \quad \Psi(x) \equiv \mathrm{e}^{i \phi(x)}$ and the integral of the screening charge

$$
Q(x) \equiv \Pi^{-1} \int_{x}^{2 \pi+x} \gamma(y) \Psi(y)^{2} d y
$$

Current

$$
J=\frac{k}{2 \pi} h_{L} \partial h_{L}^{-1} d x=\frac{k}{2 \pi}\left(\begin{array}{cc}
-i \partial \phi-\beta \gamma & -\partial \beta+2 i(\partial \phi) \beta+\beta^{2} \gamma \\
\gamma & i \partial \phi+\beta \gamma
\end{array}\right) d x
$$

As we see, $M_{\hat{L G}}$ may be described via classical scalar field $\phi$ and classical $\beta \gamma$-system (essentially a complex scalar field). Both may be quantized in Fock spaces. The quantum version of (7) may be produced by Wick ordering the products of free fields. One obtains this way Fock space representations of $\hat{L s l}(2)$ Kac-Moody algebra (the so called Wakimoto modules) [34. The irreducible unitary representations of $\hat{L s l}(2)$ may be realized in the cohomology of a

complex of Fock space ones, with the differential obtained from various (regularized) powers of the integral $Q$ of the screening operator [35]. The quantum version of field $h_{L}(x)$ may again be constructed by Wick-ordering (5). For the details, we refer to the original work [35]. A lattice version of this construction will be presented in Sections 7 to 9 .

\section{WZW theory on lattice}

Let us consider the lattice cylinder $\mathbf{Z} \times \mathbf{Z}$ with identification $\left(n^{+}, n^{-}\right) \cong\left(n^{+}+N, n^{-}+N\right)$ $\left(n^{+}, n^{-}\right.$are integer values of the light-cone variables $\left.x^{ \pm}\right)$. The following difference equation is the lattice counterpart of classical equation of motion (2.1) of the WZW theory:

$$
g\left(n^{+}, n^{-}\right) g\left(n^{+}+1, n^{-}\right)^{-1}=g\left(n^{+}, n^{-}-1\right) g\left(n^{+}+1, n^{-}-1\right)^{-1} .
$$

The space of solutions may be parametrized by the values $g(n, n)$ and $g(n+1, n)$ of the lattice field $g$ at two subsequent time slices (the lattice Cauchy data). The general solution has the form

$$
g\left(n^{+}, n^{-}\right)=g_{L}\left(n^{+}\right) g_{R}\left(n^{-}\right)^{-1}
$$


where

$$
g_{L, R}(n+N)=g_{L, R}(n) \gamma_{L, R}
$$

with $\gamma_{L}=\gamma_{R}$. Let $P_{L, R}^{N}$ denote the space of $g_{L, R}$ satisfying (3). Again, space of all solutions of (1) is

$$
P^{N}=\Delta / G
$$

where $\Delta$ is the submanifold of $P_{L}^{N} \times P_{R}^{N}$ given by the equation $\gamma_{L}=\gamma_{R}$. We shall consider $P_{L}^{N}$ with the Poisson bracket given by

$$
\begin{aligned}
& \left\{g_{L}(n)_{1}, g_{L}(m)_{2}\right\}=g_{L}(n)_{1} g_{L}(m)_{2} r^{ \pm} \\
& \left\{g_{L}(n)_{1}, g_{L}(n)_{2}\right\}=r^{+} g_{L}(n)_{1} g_{L}(n)_{2}+g_{L}(n)_{1} g_{L}(n)_{2} r^{-} \text {, } \\
& \left\{g_{L}(n)_{1}, \gamma_{L 2}\right\}=g_{L}(n)_{1} \gamma_{L 2} r^{-}-g_{L}(n)_{1} r^{+} \gamma_{L 2} \text {, } \\
& \left\{\gamma_{L 1}, \gamma_{L 2}\right\}=r^{+} \gamma_{L 1} \gamma_{L 2}-\gamma_{L 1} r^{+} \gamma_{L 2}-\gamma_{L 2} r^{-} \gamma_{L 2}+\gamma_{L 1} \gamma_{L 2} r^{-} \text {. }
\end{aligned}
$$

where in the first line \pm corresponds to $n_{<}^{>} m,|n-m|<N . \quad P_{R}^{N}$ has the Poisson bracket of opposite sign. These two brackets induce a (local) Poisson bracket on $P^{N}$ which for the Cauchy data takes the form:

$$
\begin{array}{ll}
\left\{g(n, n)_{1}, g(n, n)_{2}\right\} & =\left[r, g(n, n)_{1} g(n, n)_{2}\right], \\
\left\{g(n, n)_{1}, g(m, m)_{2}\right\} & =0 \text { for } m \neq n \bmod N, \\
\left\{g(n+1, n)_{1}, g(n+1, n)_{2}\right\} & =\left[r, g(n+1, n)_{1} g(n+1, n)_{2}\right], \\
\left\{g(n+1, n)_{1}, g(m+1, m)_{2}\right\} & =0 \text { for } m \neq n \bmod N, \\
\left\{g(n, n)_{1}, g(n+1, n)_{2}\right\} & =-g(n, n)_{1} g(n+1, n)_{2} r^{+}, \\
\left\{g(n, n)_{1}, g(n, n-1)_{2}\right\} & =r^{-} g(n, n)_{1} g(n, n-1)_{2}, \\
\left\{g(n, n)_{1}, g(m+1, m)_{2}\right\} & =0 \text { for } m \neq n, n-1 \bmod N .
\end{array}
$$

$\underline{\text { Lattice conformal symmetry }}$

The above Poisson brackets on $P_{L, R}^{N}$ and $P^{N}$ are invariant under shifts

$$
g_{L, R}(\cdot) \stackrel{\mathcal{U}_{L, R}}{\longmapsto} g_{L, R}(\cdot+1) .
$$

In general, if $D: \mathbf{Z} \rightarrow \mathbf{Z}$ is an increasing map s. t. $D\left(n+N^{\prime}\right)=D(n)+N$ (necessarily, $\left.N^{\prime} \leq N\right)$ then it induces a Poisson map

$$
P_{L, R}^{N} \ni g_{L, R}(\cdot) \stackrel{\mathcal{D}_{L, R}}{\longmapsto} g_{L, R}(D(\cdot)) \in P_{L, R}^{N^{\prime}} .
$$

These maps, of the form of decimation, constitute the lattice versions of the conformal transformations: on the lattice the conformal invariance is expressed as invariance under a renormalization group type transformations. 
$\underline{\text { Lattice loop group symmetry }}$

$G^{N} \operatorname{acts}$ on $P_{L}^{N}$ by

$$
g_{L}(n) \mapsto h(n)^{-1} g_{L}(n)
$$

This action does not preserve the Poisson structure of $P_{L}^{N}$ but it becomes a Poisson-Lie symmetry if we consider each component of $G^{N}$ as a PL group $G_{r}$ with Poisson bracket (2.19). Let us recall that in the continuum, current $J=(k / 2 \pi) g_{L} \partial g_{L}^{-1}$ was almost the moment map for the loop group symmetry of $P_{L}$. Its Poisson brackets corresponded to the canonical bracket on the dual $\hat{L G}^{*}$ of the central extension of the loop group Lie algebra. Similarly, the lattice version of the current

$$
J(n)=g(n+1) g(n)^{-1}
$$

does not correspond to the Poisson bracket on $\left(G_{r}^{*}\right)^{N}$ as should the moment map for PL symmetry $G_{r}^{N}$ but to its deformation:

$$
\begin{aligned}
& \left\{J(n)_{1}, J(n)_{2}\right\} \quad=r^{+} J(n)_{1} J(n)_{2}+J(n)_{1} J(n)_{2} r^{-}, \\
& \left\{J(n)_{1}, J(n+1)_{2}\right\}=-J(n+1)_{2} r^{+} J(n)_{1} \text {, } \\
& \left\{J(n)_{1}, J(m)_{2}\right\} \quad=0 \quad \text { if } m \neq n-1, n, n+1 \bmod N \text {. }
\end{aligned}
$$

$G^{N}$ with the Poisson bracket (10) describes the semiclassical object which corresponds to the lattice Kac-Moody algebra which was introduced in [23, 224] and which we shall denote $\mathcal{K}_{N}$ below. We shall quantize the lattice WZW theory basing on the representation theory of $\mathcal{K}_{N}$.

$G_{r}$ Poisson-Lie symmetry

Exactly as in the continuum case, the map

$$
P_{L}^{N} \times G_{r} \ni\left(g_{L}, g\right) \mapsto g_{L} g \in P_{L}^{N}
$$

gives a PL symmetry of $P_{L}^{N}$ whose moment map is (locally) given by the lift of the monodromy $\gamma \in G$ to $\left(\gamma^{+}, \gamma^{-}\right) \in G_{r}^{*}$.

Again as in continuum, we may parametrize

$$
g_{L}(n)=h(n) \mathrm{e}^{i n \tau} g_{0}^{-1} \equiv h_{L}(n) g_{0}^{-1}
$$

where $(h, \tau)$ are coordinates of the model space $M_{\mathcal{K}_{N}}$ of the (semiclassical version of) lattice Kac-Moody algebra $\mathcal{K}_{N}$ and $\left(g_{0}, \tau\right)$ of the model space $M_{G_{r}}$ introduced above. The space of quantum states corresponding to $M_{\mathcal{K}_{N}}$ will be a discrete sum of representations of $\mathcal{K}_{N}$ and that corresponding to $P_{L}^{N}$ will be a sum of their combinations with irreducible representations of quantum group $\mathcal{U}_{q}(\mathcal{G})$. Quantum $h_{L}(n)$ will be built of vertex operators of lattice Kac-Moody 
algebra $\mathcal{K}_{N}$ and quantum chiral WZW fields $g_{L}(n)$ will combine $h_{L}(n)$ with quantum $g_{0}^{-1}$ composed of $\mathcal{U}_{q}(\mathcal{G})$ vertex (or Clebsch-Gordan) operators. The rest of the present exposition will be devoted to the realization of this program for $G=S L(2)$.

\section{Lattice Kac-Moody algebra}

The solutions $r^{ \pm}$of CYB equations may be quantized in representations, at least in some cases, to the solutions $R^{ \pm}$of the quantum version of the Yang-Baxter equation (without spectral parameter):

$$
R_{12}^{ \pm} R_{13}^{ \pm} R_{23}^{ \pm}=R_{23}^{ \pm} R_{13}^{ \pm} R_{12}^{ \pm}
$$

$R^{+}=P\left(R^{-}\right)^{-1} P$ where $P$ interchanges the factors in the tensor product and $R^{ \pm}=1+\frac{1}{i} r^{ \pm}+$ $\mathcal{O}\left(k^{-2}\right)$. This may be done for example for the standard $r$-matrix (2.17). For $S L(2)$ with which, for simplicity, we shall work from now on,

$$
R^{+}=q^{1 / 2}\left(\begin{array}{cccc}
q^{-1} & 0 & 0 & 0 \\
0 & 1 & q^{-1}-q & 0 \\
0 & 0 & 1 & 0 \\
0 & 0 & 0 & q^{-1}
\end{array}\right), \quad R^{-}=q^{-1 / 2}\left(\begin{array}{cccc}
q & 0 & 0 & 0 \\
0 & 1 & 0 & 0 \\
0 & q-q^{-1} & 1 & 0 \\
0 & 0 & 0 & q
\end{array}\right)
$$

in the fundamental representation. $q=\mathrm{e}^{\pi i /(k+2)} . S L(2)$-based lattice Kac-Moody algebra $\mathcal{K}_{N}$ [23], 24] is an associative algebra with generators organized into $2 \times 2$ matrices ( $n$ is taken modulo $N$ )

$$
\mathrm{J}(n) \equiv\left(\begin{array}{ll}
\mathrm{J}(n)_{11} & \mathrm{~J}(n)_{12} \\
\mathrm{~J}(n)_{21} & \mathrm{~J}(n)_{22}
\end{array}\right) \in \mathcal{K}_{N} \otimes \operatorname{End}\left(\mathbf{C}^{2}\right)
$$

such that

$$
\mathrm{J}(n)_{11} \mathrm{~J}(n)_{22}-q^{-1} \mathrm{~J}(n)_{21} \mathrm{~J}(n)_{12}=q^{1 / 2}
$$

and with relations

$$
\begin{array}{ll}
\mathrm{J}(n)_{1} \mathrm{~J}(n)_{2} & =R^{+} \mathrm{J}(n)_{2} \mathrm{~J}(n)_{1} R^{-}, \\
\mathrm{J}(n)_{1} \mathrm{~J}(n+1)_{2} & =\mathrm{J}(n+1)_{2}\left(R^{+}\right)^{-1} \mathrm{~J}(n)_{1}, \\
\mathrm{~J}(n)_{1} \mathrm{~J}(m)_{2} & =\mathrm{J}(m)_{2} \mathrm{~J}(n)_{1} \quad \text { for } m \neq n-1, n, n+1 .
\end{array}
$$

Notice that the semi-classical versions of those relations obtained by expanding to the leading order in $k^{-1}$ and replacing the commutator by $\frac{1}{i}$ times the Poisson bracket (of order $k^{-1}$ ) give relations (6.10). The conformal symmetry is realized on the level of lattice Kac-Moody algebras by "block spin" homomorphisms $\mathcal{D}$ corresponding to increasing maps $D: \mathbf{Z} \rightarrow \mathbf{Z}$ satisfying $D\left(n+N^{\prime}\right)=D(n)+N$ :

$$
\mathcal{K}_{N^{\prime}} \ni \mathrm{J}(n) \stackrel{\mathcal{D}}{\longmapsto} \mathrm{J}(D(n+1)-1) \cdots \mathrm{J}(D(n)+1) \mathrm{J}(D(n)) \in \mathcal{K}_{N}
$$


(Recall that Diff $+S^{1}$ acts by automorphisms on continuum Kac-Moody algebras).

\section{Free field representations of $\mathcal{K}_{N}$}

As in continuum, the lattice Kac-Moody algebra may be expressed by free fields or rather their simple deformations. The fields in question are $\Theta(n)$ (invertible) and $\mathrm{B}(n), \Gamma(n)$ which should be thought of as $\epsilon$-lattice versions of fields $\mathrm{e}^{i \epsilon \partial \phi(\epsilon n)}, \beta(\epsilon n)$ and $\gamma(\epsilon n)$. Their defining relations are:

$$
\begin{array}{ll}
\Theta(n) \Theta(n+1) & =q^{1 / 2} \Theta(n+1) \Theta(n), \\
q \mathrm{~B}(n) \Gamma(n) & =q^{-1} \Gamma(n) \mathrm{B}(n)+q-q^{-1}, \\
\Theta(n) \mathrm{B}(n) & =q \mathrm{~B}(n) \Theta(n), \\
\Theta(n) \mathrm{B}(n+1) & =q^{-1} \mathrm{~B}(n+1) \Theta(n), \\
\Theta(n) \Gamma(n) & =q^{-1} \Gamma(n) \Theta(n), \\
\Theta(n) \Gamma(n+1) & =q \Gamma(n+1) \Theta(n) .
\end{array}
$$

All other commutators are trivial. The first relation is a lattice version of the $u(1)$ current algebra. Second equation is a deformation of the local oscillator algebra. The rest introduces (essentially for convenience) a twist in the tensor product of the two algebras.

Let us assume that $k$ is integer and consequently $q^{2 p}=1$ where $p \equiv k+2$. Let us also take, for convenience, $N$ odd. Deformed free fields $\Theta, \mathrm{B}$ and $\Gamma$ may be realized in finite-dimensional space $\mathcal{H}$ with the bases $|\underline{\alpha}\rangle \otimes|\underline{s}\rangle$ where $\underline{\alpha}=\left(\alpha_{0}, \alpha_{2}, \ldots, \alpha_{N-1}\right), \alpha_{m} \in \mathbf{Z}_{4 p}, \sum \alpha_{2 m}=0$, and $\underline{s}=\left(s_{0}, s_{1}, \ldots, s_{N-1}\right), s_{n}=0,1, \ldots, p-1$. The even $\Theta(n)$ (except $\left.\Theta(N-1)\right)$ act diagonally, the odd ones by shifts of $\alpha$ 's. B $(n)$ 's are the lowering and $\Gamma(n)$ 's the raising operators for the parafermionic occupation numbers $s_{n}<p$. More exactly, the action of field operators is given by the formulae:

$$
\begin{array}{ll}
\Theta(2 m)|\underline{\alpha}\rangle \otimes|\underline{s}\rangle & =z_{2 m} q^{s_{2 m+1}-s_{2 m}+\alpha_{2 m} / 2}|\underline{\alpha}\rangle \otimes|\underline{s}\rangle \quad \text { for } 2 m \neq N-1, \\
\Theta(N-1)|\underline{\alpha}\rangle \otimes|\underline{s}\rangle & =z_{N-1} q^{s_{0}-s_{N-1}+\alpha_{N-1} / 2}\left|\underline{\alpha^{\prime}}\right\rangle \otimes|\underline{s}\rangle, \\
\Theta(2 m+1)|\underline{\alpha}\rangle \otimes|\underline{s}\rangle & =z_{2 m+1} q^{s_{2 m+2}-s_{2 m+1}}\left|\underline{\alpha^{\prime \prime}}\right\rangle \otimes|\underline{s}\rangle, \\
\mathrm{B}(n)|\underline{\alpha}\rangle \otimes|\underline{s}\rangle & =\left(1-q^{-2 s_{n}}\right)|\underline{\alpha}\rangle \otimes\left|\underline{s^{\prime}}\right\rangle \\
\Gamma(n)|\underline{\alpha}\rangle \otimes|\underline{s}\rangle & =|\underline{\alpha}\rangle \otimes\left|\underline{s^{\prime \prime}}\right\rangle \quad \text { or } 0 \text { if } s_{n}=p-1 .
\end{array}
$$

where

$$
\begin{aligned}
\underline{\alpha^{\prime}} & =\left(\alpha_{0}-1, \alpha_{2}, \ldots, \alpha_{N-3}, \alpha_{N-1}+1\right) \\
\underline{\alpha^{\prime \prime}} & =\left(\alpha_{0}, \ldots, \alpha_{2 m-2}, \alpha_{2 m}+1, \alpha_{2 m+2}-1, \alpha_{2 m+4}, \ldots, \alpha_{N-1}\right) \\
\underline{s^{\prime}} & =\left(s_{0}, \ldots, s_{n-1}, s_{n}-1, s_{n+1}, \ldots, s_{N-1}\right) \\
\underline{s^{\prime \prime}} & =\left(s_{0}, \ldots, s_{n-1}, s_{n}+1, s_{n+1}, \ldots, s_{N-1}\right) .
\end{aligned}
$$

$z_{n}$ are arbitrary non-zero complex numbers. The above formulae give irreducible representations of the free field algebra $\mathcal{F}_{N}$. They are characterized by the values of the Casimirs $\Theta(n)^{4 p}=z_{n}^{4 p}$, and $\Pi \equiv q^{-1 / 2} \Theta(N-1) \cdots \Theta(1) \Theta(0)=q^{-(N+1) / 4} \prod_{n} z_{n}$. 
Now, the crucial fact is that there exists a homomorphism of the lattice Kac-Moody algebra $\mathcal{K}_{N}$ into $\mathcal{F}_{N}$ given by the following realization of the generators $\mathrm{J}(n)$ via free fields $\Theta$, B and $\Gamma:$

$$
\begin{gathered}
\mathrm{J}(n)= \\
\left(\begin{array}{cc}
\Theta(n)+q^{-1 / 2} \Theta(n)^{-1} \mathrm{~B}(n+1) \Gamma(n) & -\Theta(n) \mathrm{B}(n)+q^{-1 / 2} \Theta(n)^{-1} \mathrm{~B}(n+1)(1-\Gamma(n) \mathrm{B}(n)) \\
q^{1 / 2} \Theta(n)^{-1} \Gamma(n) & q^{1 / 2} \Theta(n)^{-1}(1-\Gamma(n) \mathrm{B}(n))
\end{array}\right) .
\end{gathered}
$$

Expressions (3) are the lattice version of the Wakimoto realization of Kac-Moody currents (5.77). Due to homomorphism (3), each representation of algebra $\mathcal{F}_{N}$ induces a representation of $\mathcal{K}_{N}$. In particular this holds for irreducible representations of $\mathcal{F}_{N}$ in space $\mathcal{H}$ described above so that $\mathcal{H}$ becomes a $\mathcal{K}_{N}$-module. The lattice Wakimoto modules obtained this way are irreducible if $\Pi \neq q^{r}$ for integer $r$ (the generic case). For $\Pi=q^{r}$ we shall study their reducibility mimicking the cohomological constructions of [35] which extended the original work of Felder [36] on free field representations of the Virasoro algebra to the case of $\hat{L s l}(2)$ Kac-Moody algebra.

\section{Lattice Bernard-Felder cohomology}

We shall need an extension of free field algebra $\mathcal{F}_{N}$ obtained by adding the invertible generator of zero mode $\Psi(0)$ with the only nontrivial commutators

$$
\begin{array}{ll}
\Theta(0) \Psi(0) & =q^{-1 / 2} \Psi(0) \Theta(0), \\
\Theta(N-1) \Psi(0) & =q^{-1 / 2} \Psi(0) \Theta(N-1), \\
\mathrm{B}(0) \Psi(0) & =q \Psi(0) \mathrm{B}(0), \\
\Gamma(0) \Psi(0) & =q^{-1} \Psi(0) \Gamma(0) .
\end{array}
$$

With the zero mode added, we may construct the lattice free field vertex operator corresponding to continuum $\mathrm{e}^{i \phi(\epsilon n)}$ :

$$
\begin{array}{ll}
\Psi(n)=\Theta(n-1) \ldots \Theta(1) \Theta(0) \Psi(0) & \text { for } n>0 \\
\Psi(n)=\Theta(n)^{-1} \ldots \Theta(-2)^{-1} \Theta(-1)^{-1} \Psi(0) & \text { for } n<0
\end{array}
$$

Notice that

$$
\Psi(n+N)=q^{1 / 2} \Pi \Psi(n)=q^{-1 / 2} \Psi(n) \Pi .
$$

Let us group the lattice Wakimoto modules into families of $2 p$ elements with $\Theta(n)^{4 p}$ fixed and $\Pi=q^{r} Z$ with $Z$ fixed and $r=-p+1,-p+2, \ldots, p$. Within a fixed family we shall label the modules as $\mathcal{H}_{q^{r}}$ according to the value of $\Pi$. $\Psi(0)$ may be implemented (uniquely up to normalization) as an operator from $\mathcal{H}_{q^{r}}$ to $\mathcal{H}_{q^{r-1}}$. We shall introduce the lattice version of the integral of the screening charge (compare (5.6)) :

$$
Q(n)=\Pi^{-1} \sum_{m=n}^{n+N-1} \Gamma(n) \Psi(m)^{2} .
$$


$Q(n)$ is nilpotent in the free field representations we consider:

$$
Q(n)^{p}=0
$$

(this essentially follows from the same property of $\Gamma(m)$ ). As a result, we have the following complexes of vector spaces for $r=0,1, \ldots, p$ :

$$
\begin{aligned}
0 & \rightarrow \mathcal{H}_{q^{-r}} \stackrel{Q^{p-r}}{\rightarrow} \mathcal{H}_{q^{r}} \stackrel{Q^{r}}{\rightarrow} \mathcal{H}_{q^{-r}} \rightarrow 0 \\
0 & \rightarrow \mathcal{H}_{q^{r}} \stackrel{Q^{r}}{\rightarrow} \mathcal{H}_{q^{-r}} \stackrel{Q^{p-r}}{\rightarrow} \mathcal{H}_{q^{r}} \rightarrow 0
\end{aligned}
$$

The important point is that for the non-generic case when $Z=1$ (i.e. $\Pi$ is a $2 p$-th root of unity), the maps of the complexes are independent of the argument $n$ of $Q$ and commute with currents $L(n)$ so that we obtain complexes of $\mathcal{K}_{N}$-modules! These complexes have no middle cohomology. We conjecture that the remaining cohomology

and

$$
\mathcal{H}_{q^{p-r}} \supset \operatorname{ker} Q^{p-r} \cong \mathcal{H}_{q^{r}} / \operatorname{im} Q^{p-r}
$$

$$
\mathcal{H}_{q^{r}} \supset \operatorname{ker} Q^{r} \cong \mathcal{H}_{q^{-r}} / \operatorname{im} Q^{r}
$$

gives irreducible representations of $\mathcal{K}_{N}$.

\section{Vertex operators}

Up to now, we have considered only lattice currents. We shall also need lattice WZW vertex operators corresponding to classical continuum fields $h_{L}(x)$ on model space $M_{\hat{L G}}$ of $\hat{L}$ sl (2) Kac-Moody group. We put (compare (5.5))

$$
h_{L}(n)=\left(\begin{array}{cc}
\left(\Pi-\Pi^{-1}\right) \Psi(n)+\mathrm{B}(n) \Psi(n)^{-1} Q(n) & \mathrm{B}(n) \Psi(n)^{-1} \\
\Psi(n)^{-1} Q(n) & \Psi(n)^{-1}
\end{array}\right) .
$$

(we have omitted matrix $\left(\begin{array}{cc}\left(\Pi-\Pi^{-1}\right)^{-1} & 0 \\ 0 & 1\end{array}\right)$ on the right hand side of (5.5) since it becomes singular for $\Pi= \pm 1$ ). Two raws of $h_{L}(n)$ correspond to the values $\pm 1 / 2$ of the magnetic number of the WZW vertex operator in spin $1 / 2$ representation. The vertex operators of higher spins are polynomials of those in fundamental representation. $h_{L}(n)$ have twisted periodicity

$$
h_{L}(n+N)=q^{-1 / 2} h_{L}(n)\left(\begin{array}{cc}
\Pi & 0 \\
0 & \Pi^{-1}
\end{array}\right) .
$$

They are related to the currents by

$$
h_{L}(n+1)=\mathrm{J}(n) h_{L}(n)
$$

and have with them the following commutation relations:

$$
\mathrm{J}(n)_{1} R^{-} h_{L}(n)_{2}=h_{L}(n)_{2} \mathrm{~J}(n)_{1},
$$




$$
\begin{array}{ll}
\mathrm{J}(n)_{1} h_{L}(n+1)_{2} & =R^{+} h_{L}(n+1)_{2} \mathrm{~J}(n)_{1}, \\
\mathrm{~J}(n)_{1} h_{L}(m)_{2} & =h_{L}(m)_{2} \mathrm{~J}(n)_{1} \quad \text { for } m \neq n, n+1
\end{array}
$$

Clearly, $h_{L}(n)$ 's act in $\bigoplus_{r=-p+1}^{p} \mathcal{H}_{q^{r}}$. For the generic case when $\Pi$ is not a $2 p$-th root of unity, the commutation relations of $h_{L}(n)$ 's are given by

$$
\begin{aligned}
h_{L}(n)_{1} h_{L}(m)_{2} & =h_{L}(m)_{2} h_{L}(n)_{1} D^{ \pm}(\Pi) \quad \text { for } n_{<}^{>} m, \quad|n-m|<N, \\
h_{L}(n)_{1} h_{L}(n)_{2} & =R^{ \pm} h_{L}(n)_{2} h_{L}(n)_{1} D^{\mp}(\Pi)
\end{aligned}
$$

where the braiding matrices

$$
\begin{aligned}
& D^{ \pm}(\Pi)= \\
& q^{\mp 1 / 2}\left(\begin{array}{cccc}
1 & & & 0 \\
0 & 1 \pm\left(q^{ \pm 2}-1\right) \Pi^{ \pm 1}\left(\Pi-\Pi^{-1}\right)^{-1} & \pm\left(q^{ \pm 2}-1\right) \Pi^{\mp 1}\left(\Pi-\Pi^{-1}\right)^{-1} & 0 \\
0 & \mp\left(q^{ \pm 2}-1\right) \Pi^{ \pm 1}\left(\Pi-\Pi^{-1}\right)^{-1} & 1 \mp\left(q^{ \pm 2}-1\right) \Pi^{\mp 1}\left(\Pi-\Pi^{-1}\right)^{-1} & 0 \\
0 & 0 & 0 & 1
\end{array}\right)
\end{aligned}
$$

and coincide with special $6 j$-symbols of quantum group $\mathcal{U}_{q}(\operatorname{sl}(2))$ [37.

For the non-generic case $Z=1$, the raws of $h_{L}(n)$ induce the following maps between exact sequences of vector spaces:

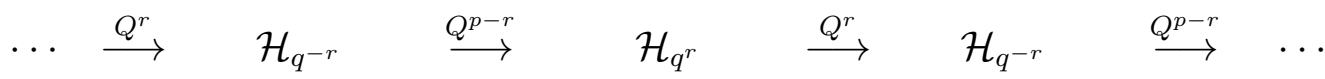

$$
\begin{aligned}
& \downarrow h_{L}(n)_{a 1} \quad \downarrow h_{L}(n)_{a 2} \quad \downarrow h_{L}(n)_{a 1} \\
& \ldots \stackrel{Q^{r+1}}{\longrightarrow} \mathcal{H}_{q^{-r-1}} \quad \stackrel{Q^{p-r-1}}{\longrightarrow} \quad \mathcal{H}_{q^{r+1}} \quad \stackrel{Q^{r+1}}{\longrightarrow} \quad \mathcal{H}_{q^{-r-1}} \stackrel{Q^{p-r-1}}{\longrightarrow} \quad \ldots
\end{aligned}
$$

for $r=0,1, \ldots, p-1$ and

$$
\begin{aligned}
& \ldots \stackrel{Q^{r}}{\longrightarrow} \quad \mathcal{H}_{q^{-r}} \quad \stackrel{Q^{p-r}}{\longrightarrow} \quad \mathcal{H}_{q^{r}} \quad \stackrel{Q^{r}}{\longrightarrow} \quad \mathcal{H}_{q^{-r}} \quad \stackrel{Q^{p-r}}{\longrightarrow} \quad \ldots \\
& \downarrow h_{L}(n)_{a 2} \quad \downarrow h_{L}(n)_{a 1} \quad \downarrow h_{L}(n)_{a 2} \\
& \ldots \stackrel{Q^{r-1}}{\longrightarrow} \mathcal{H}_{q^{-r+1}} \quad \stackrel{Q^{p-r+1}}{\longrightarrow} \quad \mathcal{H}_{q^{r-1}} \quad \stackrel{Q^{r-1}}{\longrightarrow} \quad \mathcal{H}_{q^{-r+1}} \quad \stackrel{Q^{p-r+1}}{\longrightarrow} \ldots
\end{aligned}
$$

for $r=1,2, \ldots, p$. All squares in the above diagrams are commutative! Define for $r=$ $0,1, \ldots, p$

$$
\begin{array}{rlrl}
\mathcal{H}_{q^{r}}^{\prime} & \equiv \operatorname{ker} Q^{r} \subset \mathcal{H}_{q^{r}}, & \mathcal{H}_{q^{-r}}^{\prime} \equiv \mathcal{H}_{q^{-r}} / \operatorname{im} Q^{r}, \\
\mathcal{H}_{q^{r}}^{\prime \prime} \equiv \mathcal{H}_{q^{r}} / \operatorname{im} Q^{p-r}, & \mathcal{H}_{q^{-r}}^{\prime \prime} \equiv \operatorname{ker} Q^{p-r} \subset \mathcal{H}_{q^{-r}}
\end{array}
$$


(notice the consistency of the definitions). These are the conjectured irreducible $\mathcal{K}_{N}$-modules at $\Pi$ a $2 p$-th root of unity. Notice that $\mathcal{H}_{q^{0}}^{\prime}=\mathcal{H}_{q^{p}}^{\prime \prime}=\{0\}$. Tracing diagrams (7) and (8) one may easily see that fields $h_{L}(n)$ act in spaces

$$
\bigoplus_{r=-p+1}^{p} \mathcal{H}_{q^{r}}^{\prime} \quad \text { and } \quad \bigoplus_{r=-p+1}^{p} \mathcal{H}_{q^{r}}^{\prime \prime}
$$

These (isomorphic) spaces quantize the lattice model space $M_{\mathcal{K}_{N}}$.

\section{Free field approach to $\mathcal{U}_{q}(\operatorname{sl}(2))$}

Lattice Kac-Moody algebra $\mathcal{K}_{N}$ is a close relative of the quantum group $\mathcal{U}_{q}(\operatorname{sl}(2))$ with generators $q^{ \pm 2 S^{3}}, S^{ \pm}$and relations

$$
q^{2 S^{3}} S^{ \pm} q^{-2 S^{3}}=q^{ \pm 2} S^{ \pm}, \quad\left[S^{+}, S^{-}\right]=\left(q^{2 S^{3}}-q^{-2 S^{3}}\right) /\left(q-q^{-1}\right) .
$$

Semi-classically, it was a deformation of the $N$-th power of the Poisson-Lie group $S L(2)_{r}^{*}$ and $S L(2)_{r}^{*}$ is a semi-classical version of $\mathcal{U}_{q}(\operatorname{sl}(2))$. In fact, $\mathcal{K}_{N}$ for $N$ changing from 1 to $\infty$ interpolates between the quantum group $\mathcal{U}_{q}(s l(2))$ and the continuum Kac-Moody algebra $\hat{L s l}(2)$ [23]. It is then no surprise that we may realize representation theory of $\mathcal{U}_{q}(\operatorname{sl}(2))$ by deformed oscillators

$$
q \mathrm{~B} \Gamma-q^{-1} \Gamma \mathrm{B}=q-q^{-1} .
$$

(this is in fact nothing else that the realization in terms difference operators [38]). All the algebraic constructions of Sections 7 to 9 may be repeated in this simplified context which may serve as a good introduction to the free field realization of $\mathcal{K}_{N}$. Matrix

$$
\gamma=q\left(\begin{array}{cc}
\Pi(1-\mathrm{B} \Gamma) & -\left(\Pi-\Pi^{-1}\right) \mathrm{B}+\Pi \mathrm{B} \Gamma \mathrm{B} \\
-\Pi \Gamma & \Pi^{-1}+\Pi \Gamma \mathrm{B}
\end{array}\right)
$$

where $\Pi, \Pi^{-1}$ commute with $B$ and $\Gamma$, satisfies the quantized version of the relations $(2.21)$ :

$$
\gamma_{1} R^{+} \gamma_{2}\left(R^{-}\right)^{-1}=R^{+} \gamma_{2}\left(R^{-}\right)^{-1} \gamma_{1}
$$

These in fact are nothing else but relations (1) in disguise as may be seen by identifying $\gamma$ with the matrix

$$
\left(\begin{array}{cc}
q^{2 S^{3}} & q\left(q-q^{-1}\right) S^{+} \\
\left(q-q^{-1}\right) S^{-} q^{2 S^{3}} & q\left(q-q^{-1}\right)^{2} S^{-} S^{+}+q^{-2 S^{3}}
\end{array}\right) .
$$

$\mathrm{B}$ and $\Gamma$ may be represented in $p$-dimensional space $\mathcal{V}$ with bases $|\sigma\rangle, \sigma=0,1, \ldots, p-1$, as parafermionic annihilation and creation operators by

$$
\mathrm{B}|\sigma\rangle=\left(1-q^{-2 \sigma}\right)|\sigma-1\rangle, \quad \Gamma|\sigma\rangle=|\sigma+1\rangle \quad \text { or } \quad 0 \quad \text { if } \quad \sigma=p-1
$$

Via (3), we then obtain a p-dimensional highest-lowest weight $\mathcal{U}_{q}(\operatorname{sl}(2))$-module. $\Pi$ acts in it as multiplication by a non-zero number. If $\Pi$ is not a $2 p$-th root of unity (the generic situation) then the module is irreducible. In general, it is only indecomposable. 
We shall again group the $\mathcal{U}_{q}(\operatorname{sl}(2))$-modules into families of $2 p$ elements $\mathcal{V}_{q^{r}}$ with $\Pi=q^{r} Z$, $r=-p+1,-p+2, \ldots, p$. Let us also introduce additional generator $\psi$ s.t.

$$
\psi \Pi=q \Pi \psi, \quad \psi \mathrm{B}=q \mathrm{~B} \psi, \quad \psi \Gamma=q^{-1} \Gamma \psi
$$

and $\mathcal{Q} \equiv \Pi \Gamma \psi^{-2}$. $\psi$ and $\mathcal{Q}$ may be implemented in $\bigoplus_{r=-p+1}^{p} \mathcal{V}_{q^{r}}$ and then $\mathcal{Q}^{p}=0$. In the non-generic case when $Z=1$, we obtain complexes of $\mathcal{U}_{q}(s l(2))$-modules

$$
\begin{aligned}
0 & \rightarrow \mathcal{V}_{q^{-r}} \stackrel{\mathcal{Q}^{r}}{\rightarrow} \mathcal{V}_{q^{r}} \stackrel{\mathcal{Q}^{p-r}}{\rightarrow} \mathcal{V}_{q^{-r}} \rightarrow 0 \\
0 & \rightarrow \mathcal{V}_{q^{r}} \stackrel{\mathcal{Q}^{p-r}}{\rightarrow} \mathcal{V}_{q^{-r}} \stackrel{\mathcal{Q}^{r}}{\rightarrow} \mathcal{V}_{q^{r}} \rightarrow 0
\end{aligned}
$$

exact in the middle. Let us denote the remaining cohomology by

$$
\begin{aligned}
\mathcal{V}_{q^{r}}^{\prime} \equiv \operatorname{ker} \mathcal{Q}^{p-r} \subset \mathcal{V}_{q^{r}}, & \mathcal{V}_{q^{-r}}^{\prime} \equiv \mathcal{V}_{q^{-r}} / \operatorname{im} \mathcal{Q}^{p-r} \\
\mathcal{V}_{q^{r}}^{\prime \prime} \equiv \mathcal{V}_{q^{r}} / \operatorname{im} \mathcal{Q}^{r}, & \mathcal{V}_{q^{-r}}^{\prime \prime} \equiv \operatorname{ker} \mathcal{Q}^{r} \subset \mathcal{V}_{q^{-r}}
\end{aligned}
$$

for $r=0,1, \ldots, p$. Notice that $\mathcal{V}_{q^{p}}^{\prime}=\mathcal{V}_{q^{0}}^{\prime \prime}=\{0\} . \quad \mathcal{V}_{q^{r}}^{\prime} \cong \mathcal{V}_{q^{r}}^{\prime}$ give the irreducible highest weight representations of $\mathcal{U}_{q}(\operatorname{sl}(2))$ with spin $S=-r / 2-1 / 2$ (i.e. the highest weight vector is the eigenvector of $q^{2 S^{3}}$ with eigenvalue $q^{2 S}$ ). Similarly, $\mathcal{V}_{q^{r}}^{\prime \prime} \cong \mathcal{V}_{q^{-r}}^{\prime \prime}$ give the irreducible highest weight representations of $\mathcal{U}_{q}(s l(2))$ of spin $r / 2-1 / 2$.

$\mathcal{U}_{q}(\operatorname{sl}(2))$ vertex operators are

$$
g_{0}=\left(\begin{array}{cc}
-\left(\Pi-\Pi^{-1}\right) \psi^{-1}+\mathrm{B} \psi \mathcal{Q} & B \psi \\
\psi \mathcal{Q} & \psi
\end{array}\right)
$$

and satisfy

$$
\gamma g_{0}=q^{2} g_{0}\left(\begin{array}{cc}
\Pi & 0 \\
0 & \Pi^{-1}
\end{array}\right)
$$

compare to the semiclassical relation (4.2). They act in $\bigoplus_{r=-p+1}^{p} \mathcal{V}_{q^{r}}$ and, for generic case, satisfy the commutation relations

$$
\left(g_{0}\right)_{1}\left(g_{0}\right)_{2}=R^{ \pm}\left(g_{0}\right)_{2}\left(g_{0}\right)_{1} D^{ \pm}(\Pi)^{-1} .
$$

or, for inverse matrices

$$
\begin{gathered}
g_{0}^{-1}=\left(\begin{array}{cc}
-\psi & q \mathrm{~B} \psi \\
\psi \mathcal{Q} & q\left(\Pi-\Pi^{-1}\right) \psi^{-1}-q \mathrm{~B} \psi \mathcal{Q}
\end{array}\right)\left(\Pi-\Pi^{-1}\right)^{-1}, \\
\left(g_{0}^{-1}\right)_{1}\left(g_{0}^{-1}\right)_{2}=D^{ \pm}(\Pi)^{-1}\left(g_{0}^{-1}\right)_{2}\left(g_{0}^{-1}\right)_{1} R^{ \pm} .
\end{gathered}
$$

Also

or

$$
\left(g_{0}\right)_{1} \gamma_{2}=R^{+} \gamma_{2}\left(R^{-}\right)^{-1}\left(g_{0}\right)_{1}
$$




$$
\left(g_{0}^{-1}\right)_{1} R^{+} \gamma_{2}=\gamma_{2}\left(g_{0}^{-1}\right)_{1} R^{-}
$$

For the non-generic case $Z=1$, raws of $g_{0}$ define maps between exact sequences

$$
\begin{aligned}
& \ldots \quad \stackrel{\mathcal{Q}^{r}}{\longrightarrow} \quad \mathcal{V}_{q^{r}} \quad \stackrel{\mathcal{Q}^{p-r}}{\longrightarrow} \quad \mathcal{V}_{q^{-r}} \quad \stackrel{\mathcal{Q}^{r}}{\longrightarrow} \quad \mathcal{V}_{q^{-r}} \quad \stackrel{\mathcal{Q}^{p-r}}{\longrightarrow} \ldots \\
& \downarrow\left(g_{0}\right)_{a 1} \quad \downarrow\left(g_{0}\right)_{a 2} \quad \downarrow\left(g_{0}\right)_{a 1} \\
& \cdots \stackrel{\mathcal{Q}^{r+1}}{\longrightarrow} \quad \mathcal{V}_{q^{r+1}} \quad \stackrel{\mathcal{Q}^{p-r-1}}{\longrightarrow} \quad \mathcal{V}_{q^{-r-1}} \quad \stackrel{\mathcal{Q}^{r+1}}{\longrightarrow} \mathcal{V}_{q^{r+1}} \stackrel{\mathcal{Q}^{p-r-1}}{\longrightarrow} \ldots
\end{aligned}
$$

for $r=0,1, \ldots, p-1$ and

$$
\begin{aligned}
& \cdots \quad \stackrel{\mathcal{Q}^{r}}{\longrightarrow} \quad \mathcal{V}_{q^{r}} \quad \stackrel{\mathcal{Q}^{p-r}}{\longrightarrow} \quad \mathcal{V}_{q^{-r}} \quad \stackrel{\mathcal{Q}^{r}}{\longrightarrow} \quad \mathcal{V}_{q^{r}} \quad \stackrel{\mathcal{Q}^{p-r}}{\longrightarrow} \ldots \\
& \downarrow\left(g_{0}\right)_{a 2} \quad \downarrow\left(g_{0}\right)_{a 1} \quad \downarrow\left(g_{0}\right)_{a 2} \\
& \ldots \stackrel{\mathcal{Q}^{r-1}}{\longrightarrow} \quad \mathcal{V}_{\mathcal{Q}^{r-1}} \quad \stackrel{\mathcal{Q}^{p-r+1}}{\longrightarrow} \quad \mathcal{V}_{\mathcal{Q}^{-r+1}} \quad \stackrel{\mathcal{Q}^{r-1}}{\longrightarrow} \mathcal{V}_{\mathcal{Q}^{r-1}} \stackrel{\mathcal{Q}^{p-r+1}}{\longrightarrow} \ldots
\end{aligned}
$$

for $r=1,2, \ldots, p$ and, consequently, $g_{0}$ may be defined as an operator acting in spaces

$$
\bigoplus_{r=-p+1}^{p} \mathcal{V}_{q^{r}}^{\prime} \quad \text { and } \quad \bigoplus_{r=-p+1}^{p} \mathcal{V}_{q^{r}}^{\prime \prime}
$$

It gives there the quantum $3 j$-symbols for tensor multiplication with the fundamental representation of $\mathcal{U}_{q}(s l(2))$. Matrix elements of $g_{0}^{-1}$ act in similar spaces but with $\mathcal{V}_{q^{0}}^{\prime}$ and $\mathcal{V}_{q^{p}}^{\prime \prime}$ excluded from the direct sums (due to the factor $\left(\Pi-\Pi^{-1}\right)^{-1}$ on the right hand side of (13) ).

\section{Quantization of lattice chiral WZW model}

From the classical expression (4.1) for the vertex-IRF transformation, we should expect that the quantum field

$$
g_{L}(n)=h_{L}(n) g_{0}^{-1}
$$

composed of the vertex operators of lattice Kac-Moody algebra $\mathcal{K}_{N}$ and of quantum group $\mathcal{U}_{q}(\operatorname{sl}(2))$ should give the lattice quantization of the classical chiral field $g_{L}$ of the WZW model. Notice that this field acts on the diagonal product space

$$
\bigoplus_{r=-p+1}^{p} \mathcal{H}_{q^{r}} \otimes \mathcal{V}_{q^{r}}
$$


in the generic case when $\Pi$ is not $2 p$-th root of unity and that

$$
g_{L}(n+N)=q^{-3 / 2} g_{L}(n) \gamma,
$$

see (10.2) and (11.11). Moreover relations (10.5), (11.14) and (11.16) immediately give the commutation relations

$$
\begin{aligned}
& g_{L}(n)_{1} g_{L}(m)_{2}=g_{L}(m)_{2} g_{L}(n)_{1} R^{ \pm} \quad \text { for } n_{<}^{>} m, \quad|n-m|<N \\
& g_{L}(n)_{1} g_{L}(n)_{2}=R^{+} g_{L}(n)_{2} g(n)_{1} R^{-} \\
& g_{L}(n)_{1} R^{+} \gamma_{2}=\gamma_{2} g_{L}(n)_{1} R^{-}
\end{aligned}
$$

which, together with (11.4), quantize expressions (6.4). In particular the (quantum) vertex-IRF transformation, by combining the lattice WZW vertex operators $h_{L}(n)$ which have quantum $6 j$-symbols as braiding matrices with quantum $3 j$-symbols $g_{0}^{-1}$, produced chiral fields $g_{L}(n)$ with $R^{ \pm}$braiding matrices realizing on the lattice the program of [17].

In the non-generic case when $\Pi=q^{r}$ in $\mathcal{H}_{q^{r}}$ and $\mathcal{V}_{q^{r}}$, there is a problem in defining the right hand side of (3) because of the $(\Pi-\Pi)^{-1}$ factor on the right hand side of (11.13) which diverges when $\Pi= \pm 1$. Nevertheless, operators $g_{L}(n)$ are still well defined on the diagonal product spaces

$$
\bigoplus_{r=-p+1}^{-1} \mathcal{H}_{q^{r}}^{\prime} \otimes \mathcal{V}_{q^{r}}^{\prime} \cong \bigoplus_{r=1}^{p-1} \mathcal{H}_{q^{r}}^{\prime} \otimes \mathcal{V}_{q^{r}}^{\prime} \quad \text { and } \quad \bigoplus_{r=-p+1}^{-1} \mathcal{H}_{q^{r}}^{\prime \prime} \otimes \mathcal{V}_{q^{r}}^{\prime \prime} \cong \bigoplus_{r=1}^{p-1} \mathcal{H}_{q^{r}}^{\prime \prime} \otimes \mathcal{V}_{q^{r}}^{\prime \prime}
$$

since $\mathcal{H}_{q^{0}}^{\prime}=\mathcal{H}_{q^{p}}^{\prime \prime}=\mathcal{V}_{q^{p}}^{\prime}=\mathcal{V}_{q^{0}}^{\prime \prime}=\{0\} \cdot g_{L}(n)$ do not, however satisfy on these spaces the exchange relations (蛋)! The non-generic case $\Pi=q^{r}$ corresponds to the values of the momenta which appear in the unitary quantization of the continuum WZW theory [35]. There, one expects existence of continuum limit chiral fields $g_{L}(x)$ acting in the space

$$
\bigoplus_{S=1 / 2}^{k / 2} \mathcal{H}_{k, S} \otimes \mathcal{V}_{q^{2 S+1}}^{\prime \prime}
$$

where $\mathcal{H}_{k, S}$ carries the irreducible highest weight representation of Kac-Moody algebra $\hat{\operatorname{Lsl}}(2)$ of level $k$ and spin $S$ (the doubling of the spins in (5) is due to the fact that the lattice representations should give rise to either highest or lowest weight modules under specific continuum limit process). As noticed by numerous authors [39]-41, the continuum fields violate the exchange relation

$$
g_{L}(x)_{1} g_{L}(y)_{2}=g_{L}(y)_{2} g_{L}(x)_{1} R^{ \pm} \quad \text { for } x_{<}^{>} y, \quad|x-y|<2 \pi,
$$

which require a bigger space, containing also non-unitary $\hat{L s} s(2)$-modules in a rather complicated way. It would be nice to have a lattice realization of such an extended space in which (6) would still hold in the non-generic case. It would be also desirable to understand whether some unitarity conditions may be used to select the lattice quantization of the chiral WZW model.

In the quantized version with generic $\Pi$, the classical Poisson-Lie symmetries of phase space $P_{L}^{N}$ of the lattice chiral WZW model become quantum group symmetries. On one hand side 
$\mathcal{K}_{N}$ acts in the space of states by operators $\mathrm{J}(n)=g_{L}(n+1) g_{L}(n)^{-1}$ with the fields satisfying the following covariance relations:

$$
\begin{array}{ll}
\mathrm{J}(n-1)_{1} g_{L}(n)_{2} & =R^{+} g_{L}(n)_{2} \mathrm{~J}(n-1)_{1}, \\
\mathrm{~J}(n)_{1} R^{+} g_{L}(n)_{2} & =g_{L}(n)_{2} \mathrm{~J}(n)_{1}, \\
\mathrm{~J}(n)_{1} g_{L}(m)_{2} & =g(m)_{2} \mathrm{~J}(n)_{1} \quad \text { for } m \neq n-1, n .
\end{array}
$$

One may express the covariance of the fields also in the dual fashion by saying that the map

$$
g_{L}(n) \mapsto h(n)^{-1} g_{L}(n)
$$

generates a homomorphism of the algebra of fields $g_{L}(n)$ to its tensor product with the quantum group $\left(S L(2)_{q}\right)^{N}$ 42 whose matrix generators satisfying relations

$$
R^{ \pm} h(n)_{1} h(n)_{2}=h(n)_{2} h(n)_{1} R^{ \pm}
$$

quantizing Poisson brackets (2.19) and commuting for different $n$.

Similarly, $\mathcal{U}_{q}(s l(2))$ acts in the space of states via the action of $\gamma$. For the generic case, the covariance properties of the fields are expressed by (国) or, dually, by saying that the map

$$
g_{L}(n) \mapsto g_{L}(n) g
$$

generates a homomorphism of the field algebra into its tensor product with $S L(2)_{q}$.

The lattice conformal symmetry given by increasing maps $D: \mathbf{Z} \rightarrow \mathbf{Z}, D\left(n+N^{\prime}\right)=$ $D(n)+N$, induces, by decimation

$$
g_{L}(n) \mapsto g_{L}(D(n))
$$

a homomorphism of the field algebras implemented by maps between the corresponding spaces of states. It remains to understand whether some sort of lattice Sugawara construction [43] survives on the lattice (it does for the $u(1)$ case). This is the principal open problem of the lattice WZW theory.

In the above exposition, we have concentrated on the left-moving part of the WZW theory. Right-handed piece of the lattice model may be constructed in exactly the same way, replacing

$q \rightarrow q^{-1}$. The left-right space of states may then be obtained by combining the spaces of representations of the left and right lattice Kac-Moody algebras as we combined those of the left Kac-Moody algebra and those of the quantum group. We leave the details as an exercise to the reader. The quantum group symmetry of chiral models residing in the monodromy of the chiral fields disappears from the real theory which continues to possess a pair of lattice Kac-Moody symmetries.

\section{References}

[1] V. Drinfeld, in Proceedings of the International Congress of Mathematicians, Berkeley 1986, p. 798. 
[2] M. Jimbo, Lett. Math. Phys. 10 (1985) 63.

[3] V. Drinfeld, Soviet Math. Dokl. 27 (1) (1983) 68.

[4] M. Semenov-Tian-Shansky, Publ. RIMS 21 (1985) 1237.

[5] V. Guillemin and Sh. Sternberg, Symplectic Techniques in Physics, Cambridge, Cambridge University Press 1984.

[6] O. Babelon and D. Bernard, Dressing symmetries, Saclay preprint 1991.

[7] J. Verdier, Astérisque 152-153 (1988) 305.

[8] J. Lu and A. Weinstein, Journ. Diff. Geom. 31 (1990) 501.

[9] J. Lu, Multiplicative and affine Poisson structures on Lie groups, Berkeley thesis 1990.

[10] K. Gawędzki, Commun. Math. Phys. 139 (1991) 201.

[11] O. Babelon, Phys. Lett. B 215 (1988) 523.

[12] B. Blok, Phys. Lett. B 233 (1989) 359.

[13] L. Faddeev, Commun. Math. Phys. 132 (1990) 131.

[14] A. Alekseev and S. Shatashvili, Commun. Math. Phys. 133 (1990) 353.

[15] M. Chu, P. Goddard, I. Halliday, D. Olive, A. Schwimmer, Phys. Lett. B 266 (1991) 71.

[16] H. Saleur and J.-B. Zuber, Integrable lattice models and quantum groups, Saclay preprint 1990.

[17] G. Moore, K. Reshetikhin, Nucl. Phys. B 328 (1989) 557.

[18] L. Faddeev and L. Takhtadjan, in Lecture Notes in Physics Vol. 246, Berlin: Springer 1986, p. 166.

[19] A. Volkov, Theor. Math. Phys. 74 (19888) 135.

[20] O. Babelon, Phys. Lett. B 238 (1990) 234.

[21] O. Babelon and L. Bonora, Phys. Lett. B 253 (1991) 365.

[22] L. Bonora and V. Bonservizi, Quantum sl $l_{n}$ Toda field theories, SISSA-ISAS preprint 1992.

[23] A. Alekseev, L. Faddeev and M. Semenov-Tian-Shansky, Hidden quantum groups inside Kac-Moody algebras, LOMI preprint 1991.

[24] A. Alekseev, L. Faddeev, M. Semenov-Tian-Shansky and A. Volkov, The unraveling of the quantum group structure in the WZNW theory, CERN preprint 1991. 
[25] L. Faddeev, Quantum symmetry in conformal field theory by hamiltonian methods

[26] k. Gawędzki, On lattice Kac-Moody algebras, in preparation.

[27] E. Witten, Commun. Math. Phys. 121 (1989) 351.

[28] K. Gawędzki, in Constructive Quantum Field Theory II, eds. A. Wightman and G. Velo, New York: Plenum 1990, p. 89.

[29] S. Elitzur, G. Moore, A. Schwimmer, N. Seiberg, Nucl. Phys. B 326 (1989) 104.

[30] N. Reshetikhin, V. Turaev, Commun. Math. Phys. 127 (1990) 1.

[31] V. Turaev, O. Viro, State sum of 3-manifolds and quantum 6j-symbols, LOMI preprint 1990.

[32] A. Kirillov, Elements of the Theory of Representations, Berlin, Heidelberg, New York, Springer 1975.

[33] A. Tsuchiya and Y. Kanie, Adv. Stud. Pure Math. 16 (1988) 297.

[34] M. Wakimoto, Commun. Math. Phys. 104 (1986) 605.

[35] D. Bernard and G. Felder, Commun. Math. Phys. 127 (1990) 145.

[36] G. Felder, Nucl. Phys. B 317 (1989) 215.

[37] A.N. Kirillov and N. Reshetikhin, in Infinite Dimensional Lie Algebras and Groups, ed. V. Kac, World Scientific, Singapore 1989, p. 285.

[38] L. Alvarez-Gaumé, C. Gómez and G. Sierra G., Nucl. Phys. B 330 (1990) 347.

[39] J. Fröhlich and T. Kerler, On the role of quantum groups in low dimensional local quantum field theories, ETH preprint 1990.

[40] L. Hadjivanov, R. Paunov and I. Todorov, Nucl. Phys. B 356 (1991) 387.

[41] P. Furlan, A. Ganchev and V. Petkova, Nucl. Phys. B 343 (1990) 205.

[42] S. Woronowicz, Publ. RIMS 23 (1987) 117.

[43] H. Sugawara, Phys. Rev. 170 (1968) 1659. 